\title{
Local Rings and the Connection of Spin with Statistics
}

By

\author{
H. J. BORCHERS \\ Institut des Hautes Etudes Scientifiques \\ Bures-sur-Yvette (S. et O.) France
}

\begin{abstract}
The usual investigations of the connection of spin and statistics start with the hypothesis that there exist only fields either commuting or anti-commuting for spacelike separations. Starting from local rings of measurements we want to show that this hypothesis can be added without loss of generality.
\end{abstract}

\section{Introduction}

When the concept of quantum fields was introduced in the late twenties, it was an immediate generalization from systems of finite degrees of freedom that one has to introduce commutation relations for fields describing bosons. The problem of how to incorporate Pauli's exclusion principle in such a theory was solved by P. JoRDAN and E. WIGNER [1]. The answer was simple and consisted in replacing the commutator by the anti-commutator*. The connection of spin and statistics came, at that stage, as a phenomenological input into the theory.

In the late thirties, however, it was realized that we cannot quantize a given particle, say of spin $1 / 2$, with the rules belonging to Bose statistics if we want at the same time to have positive energy [3]. This meant there was a connection between the three hypotheses,

1) The fields transform in a well defined manner under the inhomogeneous Lorentz transformation.

2) The energy is positive.

3) The fields are quantized with respect either to the rules of Bose statistics or to the rules of Fermi statistics.

A more satisfactory understanding of the connection of the assumptions 1)-3) followed the discovery of the CPT-theorem $\star \star$.

Postulate 3) says something about the modes of quantization which we admit in our theory. The question arises whether these are the only possible modes or if there exist others. This question has partly been answered by giving examples where one has other commutation relations [5] (see also references in [5]).

* See e.g. [2] for a more detailed account of this part of history.

$\star \star$ The reader will find more information on this subject, with references, in [4].

Commun. math. Phys., Vol. 1 
For measurable quantities, however, it is plausible and generally accepted that they have to commute for spacelike separations. Therefore quantities which are quantized with anticommutation or other strange commutation relations are non-measurable. So it might be possible that one has some freedom in the choice of the commutation relations for such quantities.

In this paper we start only from measurable quantities and want to show that it is always sufficient to take commutation or anticommutation relations for non-measurable fields. In other words, the assumption 3 ) which is used for the investigations of the connection of spin with statistics can be added without loss of generality.

We do not want to study here the question under what conditions we can introduce strange commutation relations. Our investigation seems to indicate that this is only the case when the theory has incidentally a higher symmetry than we can derive from our assumptions. In the examples of free ,parafields" this symmetry is a mass-degeneracy [6].

In our investigation we only use the translation invariance of the theory and the spectrum condition. It is questionable whether these assumptions are already sufficient to derive the CTP-theorem. But we will show that these assumptions allow us to derive one of the main conclusions of the CTP-theorem, namely that the masses of the particles and anti-particles are the same.

\section{Assumptions}

Assume we have assigned to every open bounded region $\mathcal{O}$ in the Minkowski space a $C^{*}$-algebra $\mathfrak{A}(\mathcal{O})$ with the properties

A 1) $\mathfrak{A}(\mathcal{O})$ is a $C^{*}$-algebra.

A 2) Let $\mathcal{O}_{1} \subset \mathcal{O}_{2}$; then $\mathfrak{A}\left(\mathcal{O}_{1}\right) \subset \mathfrak{A}\left(\mathcal{O}_{2}\right)$.

A 3) If two regions $\mathcal{O}_{1}$ and $\mathcal{O}_{2}$ are spacelike separated, then the algebras $\mathscr{A}\left(\mathcal{O}_{1}\right)$ and $\mathfrak{A}\left(\mathcal{O}_{2}\right)$ commute.

A 4) If $\left\{\mathcal{O}_{n}\right\}$ is any covering of the Minkowski space by bounded open regions, then the smallest $C^{*}$-algebra $\mathfrak{A}(\mathscr{M})$ is independent of the covering.

A 5) $\mathfrak{A}(\mathscr{M})$ contains no central elements.

Furthermore, we want to consider the translation group and we assume

T 1) To every element $a$ of the translation group there exists an automorphism $\phi(a)$ of the algebra $\mathfrak{A}(\mathscr{M})$.

T 2) $\mathfrak{A}(\mathcal{O})$ and $\mathfrak{A}(\mathcal{O}+a)$ are related by $\phi(a) \mathfrak{A}(\mathcal{O})=\mathfrak{A}(\mathcal{O}+a)$ for every bounded open region $\mathcal{O}$.

In addition to these purely algebraic assumptions we want to consider representations of this algebra:

R 1) We have $N$ different representations $D_{i}, i=1 \ldots N$ of the algebra $\mathfrak{A}(\mathscr{M})$ in the Hilbert space $\mathfrak{G}_{i}$. 
R 2) Each representation $D_{i}(\mathfrak{A}(\mathscr{M}))$ is weakly dense in $\mathscr{L}\left(\mathfrak{S}_{i}\right)$, the ring of all bounded operators in $\mathfrak{G}_{i}$.

R 3) The translations $\phi(a)$ are unitarily implementable.

If $b \in \mathfrak{U}(\mathscr{M})$, then

$$
D_{i}(\phi(a) b)=\mathscr{U}_{i}(a) D_{i}(b) \mathscr{U}_{i}^{-1}(a) .
$$

R 4) The representations $\mathscr{U}_{i}(a)$ in $\mathfrak{S}_{i}$ of the translation group fulfil the spectrum condition.

R 5) None of the Hilbert space contains a vacuum state.

In an earlier paper [7] we showed that to each representation $D$ of $\mathfrak{A}(\mathscr{M})$ fulfilling the requirements $R 1$ ) to $R 5$ ) there is associated in a canonical way a representation $D_{0}$ in a Hilbert space with vacuum. This representation is locally unitarily equivalent to the given representation. We want to assume

R 6) The representations $D_{i_{0}}$ associated with $D_{i}$ coincide.

Since we have assumed that all the representations are locally unitarily equivalent, the topology on the rings $\mathfrak{A}(\mathcal{O})$ for bounded open regions is completely irrelevant. This means we are allowed to assume

R 7) For every representation $D_{i}$ and all bounded open regions $\mathcal{O}$ the algebra $D_{i}(\mathfrak{H}(\mathcal{O}))$ is a von Neumann algebra.

This assumption will simplify our investigations. We have to make one more assumption which is the weakest form of the duality assumption:

R 8) For every bounded open region $\mathcal{O}$ and every representation we have

$$
D_{i}(\mathfrak{U}(\mathcal{O}))^{\prime} \cap D_{i}(\mathfrak{A}(\mathscr{M})) \text { is dense in } \quad D_{i}(\mathfrak{A}(\mathcal{O}))^{\prime}
$$

in the weak operator topology.

We believe that $R$ ) is already a consequence of the other assumptions, but, up to now, we have not found the proof for it.

\section{Some Lemmas}

III - 1. Lemma. Let $D$ be the representation fulfilling our assumptions. Assume $D_{0}$ is the vacuum representation associated with $D$ in a canonical way. Denote by $\phi$ the map $D \stackrel{\phi}{\longrightarrow} D_{0}$. Then for every bounded open region $\mathcal{O}$ there exists an isomorphism $\psi$ mapping

$$
D(\mathfrak{A}(\mathcal{O}))^{\prime} \stackrel{\psi}{\longrightarrow} D_{0}(\mathfrak{A}(\mathcal{O}))^{\prime}
$$

such that $\phi=\psi$ on $D(\mathfrak{U}(\mathcal{O}))^{\prime} \cap D(\mathfrak{U}(\mathscr{M}))$. Furthermore, the map $\psi$ is unitarily implementable.

Proof. In Theorem 13 of [7] we proved that for every subalgebra $\mathfrak{A} \subset \mathfrak{A}(\mathscr{M})$, there exists an $\mathcal{O}$ with $D(\mathfrak{U}(\mathcal{O})) \subset D(\mathfrak{A})^{\prime}$ having the property that the map $\phi: D(\mathfrak{I}) \stackrel{\phi}{\longrightarrow} D_{0}(\mathfrak{U})$ is continuous in the ultrastrong topo- 
logy and normal. Although we did not state it in this way it is easy to check that the proof is also valid in this situation. Hence the mapping

$$
D(\mathfrak{A}(\mathcal{O}))^{\prime} \cap D(\mathfrak{H}(\mathscr{M})) \stackrel{\phi}{\longrightarrow} D_{0}(\mathfrak{A}(\mathcal{O}))^{\prime} \cap D_{0}(\mathfrak{A}(\mathscr{M}))
$$

extends to the corresponding von Neumann algebra and is normal. Let us call $\psi$ the extension to the von Neumann algebra; then we have by assumption $\mathrm{R}$ 8) that the mapping

$$
D(\mathfrak{A}(\mathcal{O}))^{\prime} \stackrel{\psi}{\longrightarrow} D_{0}(\mathfrak{A}(\mathcal{O}))^{\prime}
$$

exists and is normal. By Corollary 20 of [7] it follows that $\psi$ is unitarily implementable. q.e.d.

III - 2. Lemma. Let us denote by $F(\mathcal{O})$ the set of all unitary operators $V$ establishing the $\operatorname{map} \psi$ from $D(\mathfrak{A}(\mathcal{O}))^{\prime} \stackrel{\psi}{\longrightarrow} D_{0}(\mathfrak{A}(\mathcal{O}))^{\prime}$; this means

$$
D_{0}(\mathfrak{A}(\mathcal{O}))^{\prime}=V D(\mathfrak{A}(\mathcal{O}))^{\prime} V^{-1} .
$$

Then $V_{1} V_{2}^{-1}$ resp. $V_{1}^{-1} V_{2}$ runs through all unitary operators of $D_{0}(\mathcal{O})$ $=D_{0}(\mathfrak{Q}(\mathcal{O}))$ resp. $D(\mathcal{O})=D(\mathfrak{A}(\mathcal{O}))$ when $V_{1}$ and $V_{2}$ run through $F(\mathcal{O})$.

Proof. Is $U$ an unitary operator from $D_{0}(\mathcal{O})$, then with $V$ in $F(\mathcal{O})$ we have also $U V$ in $F(\mathcal{O})$. Hence

$$
U V \cdot V^{-1}=U
$$

is in $D_{0}(\mathcal{O})$. This means $\left(F(\mathcal{O}) \cdot F^{-1}(\mathcal{O})\right)^{\prime \prime}>D_{0}(\mathcal{O})$. Let now $V_{1}$ and $V_{2}$ be from $F(\mathcal{O})$; then

$$
V_{2} V_{1}^{-1} D_{0}(\mathcal{O})^{\prime} V_{1} V_{2}^{-1}=V_{2} D(\mathcal{O})^{\prime} V_{2}^{-1}=D_{0}(\mathcal{O})^{\prime},
$$

whence $V_{1} V_{2}^{-1} \subset D_{0}(\mathcal{O})$. This means

$$
\begin{aligned}
& \left(F(\mathcal{O}) \cdot F^{-1}(\mathcal{O})\right)^{\prime \prime} \subset D_{0}(\mathcal{O}) . \\
& \left(F(\mathcal{O}) \cdot F^{-1}(\mathcal{O})\right)^{\prime \prime}=D_{0}(\mathcal{O}) .
\end{aligned}
$$

In the similar manner we can show

$$
\left(F^{-1}(\mathcal{O}) F(\mathcal{O})\right)^{\prime \prime}=D(\mathcal{O}), \quad \text { q.e.d. }
$$

\section{Introduction of one charged field}

In this section we assume there is given only one representation $D_{1}$. We want to show that we can introduce a "charged field" $\psi$ such that $D_{0}$ resp. $D_{1}$ appears as the representation of $\mathfrak{A}(\mathscr{M})$ in the "charge sector" zero resp. one.

Let us assign to every $V \in F(\mathcal{O})$ an abstract element $\psi(V)$ and denote by $\mathfrak{B}$ the free algebra generated by all elements of $\mathfrak{A}(\mathscr{M})$, all $\psi(V)$ and all $\psi^{*}(V)$. Let us define a functional on $\mathfrak{B}$ by the following procedure: 
1) Let $M$ be a monomial and $N(\psi)$ be the number of $\psi$ 's and $N\left(\psi^{*}\right)$ the number of $\psi^{*}$ 's in $M$. Then we define

$$
\omega(M)=0 \text { if } N(\psi)-N\left(\psi^{*}\right) \neq 0 .
$$

2) If $M$ is an element of $\mathscr{A}(\mathscr{M})$, we put $\omega(M)=\left(\Omega, D_{0}(M) \Omega\right)$, where $D_{0}$ is the representation of $\mathscr{A}(\mathscr{M})$ in the vacuum sector and $\Omega$ is the vacuum state.

3) If $N(\psi)-N\left(\psi^{*}\right)=0$, then we want to identify $M$ with an element in $\mathfrak{A}$. We do this in the following manner: We replace every element $a \in \mathfrak{A}(\mathscr{M})$ by its representative $D_{0}(a)$, every $\psi(V)$ by $V \phi^{-1}$ and every $\psi^{*}(V)$ by $\phi V^{-1}$. Here $\phi$ is the isomorphism mapping the representation $D_{1}(a)$ onto the representation $D_{0}(a)$.

4) We define $\omega\left(\Sigma M_{i}\right)=\Sigma \omega\left(M_{i}\right)$.

We have to show that this procedure is well defined.

IV - 1. Lemma. Let $M$ be a monomial with $N(\psi)-N\left(\psi^{*}\right)=0$, then the substitution defined in 3) maps $M$ onto an element of $D_{0}(\mathfrak{R})$.

Proof. By induction with respect to $N(\psi)$. For $N(\psi)=0$ the assertion is trivial. Let us assume we have proved if for $N(\psi)=n$. We have to prove it for $N(\psi)=n+1$. But $M_{n+1}$ can have only one of the following three forms:

«) $M_{n+1}=M_{k} \cdot M_{(n+1-k)}$

$$
1 \leqq k \leqq n
$$

в) $M_{n+1}=a_{1} \psi\left(V_{2}\right) M_{n} \psi^{*}\left(V_{3}\right) a_{4}$

r) $M_{n+1}=a_{1} \psi^{*}\left(V_{2}\right) M_{n} \psi\left(V_{3}\right) a_{4}$

Let $S$ be the substitution operator; then we have in case $\alpha$ )

and in case $\beta$ )

$$
S\left(M_{n+1}\right)=D_{0}\left(M_{k}\right) \cdot D_{0}\left(M_{n+1-k}\right) \in D_{0}(\mathfrak{A})
$$

$$
\begin{aligned}
S\left(M_{n+1}\right) & =D_{0}\left(a_{1}\right) V_{2} \phi^{-1} D_{0}\left(M_{n}\right) \phi V_{3}^{-1} D_{0}\left(a_{4}\right) \\
& =D_{0}\left(a_{1}\right) V_{2} \phi^{-1}\left(D_{0}\left(M_{n}\right)\right) V_{3}^{-1} D_{0}\left(a_{4}\right) \\
& =D_{0}\left(a_{1}\right) V_{2} D_{1}\left(M_{n}\right) V_{3}^{-1} D_{0}\left(a_{4}\right) .
\end{aligned}
$$

If now $D_{1}\left(M_{n}\right) \in D_{1}(\mathcal{O})$ and $V_{2} \in F^{\prime}\left(\mathcal{O}_{2}\right)$ and $V_{3} \in F\left(\mathcal{O}_{3}\right)$ then, we find as a consequence of Lemma III -2 that $V_{2} D_{1}\left(M_{n}\right) V_{3}^{-1}$ is an element of $D_{0}\left(\mathcal{O} \cup \mathcal{O}_{2} \cup \mathcal{O}_{3}\right)$. Hence

Now the case $\gamma)$ :

$$
S\left(M_{n+1}\right) \in D_{0}(a) .
$$

$$
\begin{aligned}
S\left(M_{n+1}\right) & =D_{0}\left(a_{1}\right) \phi V_{2}^{-1} D_{0}\left(M_{n}\right) V_{3} \phi^{-1} D_{0}\left(a_{4}\right) \\
& =D_{0}\left(a_{1}\right) \phi\left\{V_{2}^{-1} D_{0}\left(M_{n}\right) V_{3}\right\} D_{0}\left(a_{4}\right) .
\end{aligned}
$$

If now again $D_{0}\left(M_{n}\right) \in D_{0}(\mathcal{O}), V_{2} \in F\left(\mathcal{O}_{2}\right)$ and $V_{3} \in F\left(\mathcal{O}_{3}\right)$, then we have again

$$
V_{2}^{-1} D_{0}\left(M_{n}\right) V_{3} \in D_{1}\left(\mathcal{O} \cup \mathcal{O}_{2} \cup \mathcal{O}_{3}\right)
$$


Hence $\phi\left\{V_{2}^{-1} D_{0}\left(M_{n}\right) V_{3}\right\}$ is from $D_{0}\left(\mathcal{O} \cup \mathcal{O}_{2} \cup \mathcal{O}_{3}\right)$, which means $S\left(M_{n+1}\right) \in D_{0}(\mathscr{Q})$, and the lemma is proved. This shows that $\omega$ is defined on all elements of the free algebra $\mathfrak{B}$.

IV - 2. Theorem. The functional $\omega$ is a one-valued linear positive functional.

Proof. $\omega$ is by definition a linear functional. We have to show that it is one-valued. Since the product between elements of $\mathfrak{A}, \psi, \psi^{*}$ is free, a relation $\sum M_{i}=0$ can only occur because of relations within $\mathfrak{A}$. But the substitution-rules 3$)$ preserve all relations in $\mathfrak{A}$; hence $\sum M_{i}=0$ implies $\sum S\left(M_{i}\right)=0$ and this means $\sum \omega\left(M_{i}\right)=0$.

We now have to demonstrate that $\omega$ is a positive functional. This means for any element $X \in \mathfrak{B}$ we have to show

$$
\omega\left(X^{*} X\right) \geqq 0 .
$$

Now $X$ is a sum over monomials $M_{j}$. To every $M_{j}$ we consider the number $n_{j}=N(\psi)-N\left(\psi^{*}\right)$. So we can write

$$
X=\sum_{k} \sum_{n_{j}=k} M_{j}=\sum_{k} X_{k} .
$$

It is now sufficient to show that all expressions of the form $\omega\left(X_{k}^{*} X_{k_{c}}\right)$ are non-negative. We do this by showing that the substitution rule 3 ) maps positive elements onto positive elements.

First let $M_{0}$ be a monomial with $N(\psi)=N\left(\psi^{*}\right)=0$. We have

$$
S\left(M_{0}^{*}\right)=D_{0}\left(M_{0}^{*}\right)=D^{*}\left(M_{0}\right)=S^{*}\left(M_{0}\right) .
$$

Assume we have proved the relation $S\left(M^{*}\right)=S^{*}(M)$ for $N(\psi)=N\left(\psi^{*}\right)=k$; then we have for $N(\psi)=N\left(\psi^{*}\right)=k+1$ again the three possibilities $\alpha), \beta), \gamma$ ) of the proof of Lemma IV - 1. In the case $\alpha$ we have

$$
S\left(\left(M_{1} M_{2}\right)^{*}\right)=S\left(M_{2}^{*}\right) \cdot S\left(M_{1}^{*}\right)=S^{*}\left(M_{1} M_{2}\right) .
$$

In the case $\beta)$ :

$$
\begin{aligned}
S\left(M_{k+1}^{*}\right) & =D_{0}\left(a_{4}^{*}\right) V_{3} \phi^{-1} D_{0}\left(M_{k}^{*}\right) \phi V_{2}^{-1} D_{0}\left(a_{1}^{*}\right) \\
& =D_{0}^{*}\left(a_{4}\right) V_{3} D_{1}^{*}\left(M_{k}\right) V_{2}^{-1} D_{0}^{*}\left(a_{1}\right) \\
& =D_{0}^{*}\left(a_{4}\right) V_{3} V_{2}^{-1} V_{2} D_{1}^{*}\left(M_{k}\right) V_{2}^{-1} D_{0}^{*}\left(a_{1}\right) .
\end{aligned}
$$

$V_{3} V_{2}^{-1}$ is unitary element of some $D_{0}(\mathcal{O})$ and its adjoint is $V_{2} V_{3}^{-1}$. Since $V_{2}$ is a unitary operator we have $V_{2} D_{1}^{*}\left(M_{k}\right) V_{2}^{-1}=\left(V_{2} D_{1}\left(M_{k}\right) V_{2}^{-1}\right)^{*}$. Hence we find

$$
\begin{aligned}
S\left(M_{k+1}^{*}\right) & =\left(D_{0}\left(a_{1}\right) V_{2} D\left(M_{k}\right) V_{2}^{-1} V_{2} V_{3}^{-1} D_{0}\left(a_{4}\right)\right)^{*} \\
& =S^{*}\left(M_{k+1}\right) .
\end{aligned}
$$

In a similar manner, we get the proof for the case $\gamma$ ). So we have for any monomial with $N(\psi)=N\left(\psi^{*}\right)$ the relation

$$
S\left(M^{*}\right)=S^{*}(M) \text {. }
$$


But this implies

$$
\omega\left(X_{0}^{*} X_{0}\right) \geqq 0 .
$$

Assume we have proved that the substitution $S$ maps expressions of the form $X_{k}^{*} X_{k}$ onto positive elements for $k=0,1, \ldots, n$. The general monomial of the form $X_{n+1}$ can be written as $X_{n} \psi X_{0}$. This means

$$
S\left(\sum_{j k} X_{0}^{j^{*}} \psi^{j^{*}} X_{n}^{j} X_{n}^{k} \psi^{k} X_{0}^{k}\right)=\sum_{j k} D_{0}\left(X_{0}^{j^{*}}\right) V^{j} D_{1}\left(X_{n}^{j^{*}} X_{n}^{k}\right) V^{k-1} D_{0}\left(X_{0}^{k}\right)
$$

The finite number of elements $X_{0}^{j}$ must be in some $D_{0}(\mathcal{O})$. Take now an $\mathcal{O}_{1}$ which is spacelike separated from $\mathcal{O}$ and a $V \in F\left(\mathcal{O}_{1}\right)$. Then we have

$$
\begin{aligned}
S\left(X_{n+1}^{*} X_{n+1}\right) & =\sum_{j k} D_{0}\left(X_{0}^{j^{*}}\right) V V^{-1} V^{j} D_{1}\left(X_{n}^{j^{*}} X_{n}^{k}\right) V^{k-1} V V^{-1} D_{0}\left(X_{0}^{k}\right) \\
& =V\left(\sum_{j k} D_{1}\left(X_{0}^{j^{*}}\right) V^{-1} V^{j} D_{1}\left(X_{n}^{j^{*}} X_{n}^{k}\right) V^{k-1} V D_{0}\left(X_{0}^{k}\right)\right) V^{-1} .
\end{aligned}
$$

By Lemma III - 2, $V^{-1} V^{j}$ are elements $a^{j^{*}}$ in some $D_{1}(\mathcal{O})$ and hence

$$
\begin{aligned}
S\left(X_{n+1}^{*} X_{n+1}\right) & =V D_{1}\left\{\sum_{j}\left(X_{0}^{j^{*}} a^{j^{*}} X_{n}^{*}\right) \sum_{k}\left(X_{n}^{k} a^{k} X_{0}^{k}\right)\right\} V^{-1} \\
& =V \phi_{0}^{-1} S\left(X_{n}^{*} X_{n}^{\prime}\right) V^{-1} .
\end{aligned}
$$

Since $S\left(X_{n}^{\prime *} X_{n}^{\prime}\right)$ is positive $\phi^{-1} S\left(X_{n}^{\prime *} X_{n}^{\prime}\right)$ is positive and hence $S\left(X_{n+1}^{*} \times\right.$ $\left.\times X_{n+1}\right)$ is a positive element. This means $\omega\left(X_{n+1}^{*} X_{n+1}\right) \geqq 0$. The proof goes in an analogous way for negative $n$. This then implies $\omega\left(X^{*} X\right) \geqq 0$ q.e.d.

We have defined on the algebra $\mathfrak{Z}$ a positive linear functional. If we restrict this functional $\omega$ to the subalgebra $\mathfrak{A}$, then $\omega$ coincides with the vacuum-expectation value of the representation $D_{0}(\mathfrak{U})$. Hence $\omega$ is an extension of the vacuum functional defined on $\mathfrak{A}$ to the algebra $\mathfrak{Z}$. If we consider a fixed $\psi$ then the expressions of the form $\omega\left(\psi a \psi^{*}\right)$ are of the form $\left(V^{-1} \Omega, D_{1}(a) V^{-1} \Omega\right)$. This is again a positive functional on $\mathfrak{A}$ and coincides with the expectation value of the representation $D_{1}(\mathfrak{Q})$ with respect to the vector $V^{-1} \Omega$. Since every positive linear functional on a star algebra defines a representation [8] of this algebra, we have a representation of $\mathfrak{Z}$. This representation restricted to $\mathfrak{A}$ will be reducible and it will contain the given representations $D_{0}$ and $D_{1}$ as irreducible subrepresentations.

We will denote the representations of a again by a and those of $\psi$ again by $\psi$. We denote the set of all $\psi(V)$ such that $V$ is contained in $F(\mathcal{O})$ by $\mathfrak{F}(\mathcal{O})$.

\section{Properties of the field $\psi$}

V - 1. Proposition. For every $\psi$ we have $\psi^{*} \psi=\psi \psi^{*}=1$.

Proof. Since the representation is defined by a positive functional, it is sufficient to show for all $X, X^{\prime} \in \mathfrak{B}$ the relations

$$
\omega\left(X \psi^{*} \psi X^{\prime}\right)=\omega\left(X X^{\prime}\right) \quad \text { resp. } \omega\left(X \psi \psi^{*} X^{\prime}\right)=\omega\left(X X^{\prime}\right) \text {. }
$$


But this is an immediate consequence of the substitution rule which defines $\omega$.

V - 2. Proposition. Let $\psi_{1}$ and $\psi_{2}$ be elements of $\mathfrak{F}(\mathcal{O})$; then $\psi_{1}^{*} \psi_{2}$ and $\psi_{2} \psi_{1}^{*}$ are elements of $\mathfrak{A}(\mathcal{O})$.

Proof. Let $\psi_{1}=\psi\left(V_{1}\right)$ and $\psi_{2}=\psi\left(V_{2}\right)$. Then, by Lemma III -2 there exists $u$ resp. $v$ from $\mathfrak{A}(\mathcal{O})$ such that

$$
V_{1}^{-1} V_{2}=D_{1}(u) \quad \text { resp. } \quad V_{2} V_{1}^{-1}=D_{0}(v) .
$$

Then, for every $X, X^{\prime}$ from $\mathfrak{B}$ we have

$$
\begin{aligned}
\omega\left(X \psi_{1}^{*} \psi_{2} X^{\prime}\right) & =\left(\Omega, S(X) \phi V_{1}^{-1} V_{2} \phi^{-1} S\left(X^{\prime}\right) \Omega\right) \\
& =\left(\Omega, S(X) \phi\left(D_{1}(u)\right) S\left(X^{\prime}\right) \Omega\right) \\
& =\left(\Omega, S(X) D_{0}(u) S\left(X^{\prime}\right) \Omega\right)=\omega\left(X u X^{\prime}\right) .
\end{aligned}
$$

In a similar way, we get

$$
\omega\left(X \psi_{2} \psi_{1}^{*} X^{\prime}\right)=\omega\left(X v X^{\prime}\right) .
$$

Since this holds for all $X$ and $X^{\prime}$ we have

$$
\psi_{1}^{*} \psi_{2}=u \text { and } \psi_{2} \psi_{1}^{*}=v \text {, q.e.d. }
$$

V - 3. Proposition. Let $\mathcal{O}_{1}$ and $\mathcal{O}_{2}$ be two spacelike separated regions. Assume $\psi \in \mathfrak{F}\left(\mathcal{O}_{1}\right)$ and $a \in \mathfrak{A}\left(\mathcal{O}_{2}\right)$; then we have $\psi a=a \psi$.

Proof. Let $\psi=\psi(V)$

$$
\begin{aligned}
\omega\left(X \psi a X^{\prime}\right) & =\left(\Omega, S(X) V \phi^{-1} D_{0}(a) S\left(X^{\prime}\right) \Omega\right) \\
& =\left(\Omega, S(X) V D_{1}(a) \phi^{-1} S\left(X^{\prime}\right) \Omega\right) \\
& =\left(\Omega, S(X) D_{0}(a) V \phi^{-1} S\left(X^{\prime}\right) \Omega\right) \\
& =\omega\left(X a \psi X^{\prime}\right) \quad \text { q.e.d. }
\end{aligned}
$$

V - 4. Lemma. Let $\mathcal{O}_{1}$ and $\mathcal{O}_{3}$ be two spacelike separated regions. Assume $\psi_{1}, \psi_{2} \in \mathfrak{F}\left(\mathcal{O}_{1}\right)$ and $\psi_{3} \in \mathfrak{F}\left(\mathcal{O}_{3}\right)$; then $\psi_{3}$ commutes with the products $\psi_{1} \psi_{2}$ and $\psi_{1}^{*} \psi_{2}^{*}$.

Proof. Assume first we have three different regions $\mathcal{O}_{i}$ which are spacelike separated from each other and $\psi_{i} \in \mathfrak{F}\left(\mathcal{O}_{i}\right)$; then we have

$$
\begin{aligned}
\psi_{1} \psi_{2} \psi_{3} & =\left(\psi_{3} \psi_{3}^{*}\right) \psi_{1} \psi_{2} \psi_{3} \\
& =\psi_{3}\left(\psi_{3}^{*} \psi_{1}\right) \psi_{2}\left(\psi_{3}\right) \\
& =\psi_{3} \psi_{2}\left(\psi_{3}^{*} \psi_{1}\right)\left(\psi_{3}\right) \\
& =\left(\psi_{3}\right)\left(\psi_{2} \psi_{3}^{*}\right) \psi_{1}\left(\psi_{3}\right) \\
& =\left(\psi_{3}\right) \psi_{1}\left(\psi_{2} \psi_{3}^{*}\right)\left(\psi_{3}\right) \\
& =\psi_{3} \psi_{1} \psi_{2} .
\end{aligned}
$$

We have used the fact that $\psi \psi^{*}=\psi^{*} \psi=1$, and that

$$
\mathfrak{F}\left(\mathcal{O}_{3}\right) \cup \mathfrak{F}\left(\mathcal{O}_{1}\right) \subset \mathfrak{F}\left(\mathcal{O}_{3} \cup \mathcal{O}_{2}\right) \text { resp. } \quad \mathfrak{F}\left(\mathcal{O}_{3}\right) \cup \mathfrak{F}\left(\mathcal{O}_{2}\right) \subset \mathfrak{F}\left(\mathcal{O}_{3} \cup \mathcal{O}_{2}\right) .
$$


This implies that we can use the propositions $\mathrm{V}-2$ and $\mathrm{V}-3$. Under the same assumption we find

$$
\psi_{1}^{*} \psi_{2}^{*} \psi_{3}=\psi_{2}^{*} \psi_{3} \psi_{1}^{*}=\psi_{3} \psi_{1}^{*} \psi_{2}^{*} .
$$

If now $\mathcal{O}_{1}=\mathcal{O}_{2}$, we choose an arbitrary region $\mathcal{O}_{4}$ which is spacelike separated to $\mathcal{O}_{1}$ as well as to $\mathcal{O}_{3}$. Let $\psi_{4} \in \mathfrak{F}\left(\mathcal{O}_{4}\right)$. Then we have

In the same manner we get

$$
\begin{aligned}
\psi_{1} \psi_{2} \psi_{3} & =\psi_{1} \psi_{4} \psi_{4}^{*} \psi_{2} \psi_{3}=\psi_{1} \psi_{4} \psi_{3} \psi_{4}^{*} \psi_{2} \\
& =\psi_{3} \psi_{1} \psi_{4} \psi_{4}^{*} \psi_{2}=\psi_{3} \psi_{1} \psi_{2} .
\end{aligned}
$$

$$
\begin{aligned}
\psi_{1}^{*} \psi_{2}^{*} \psi_{3} & =\psi_{1}^{*} \psi_{4} \psi_{4}^{*} \psi_{2}^{*} \psi_{3}=\psi_{1}^{*} \psi_{4} \psi_{3} \psi_{4}^{*} \psi_{2}^{*} \\
& =\psi_{3} \psi_{1}^{*} \psi_{4} \psi_{4}^{*} \psi_{2}^{*}=\psi_{3} \psi_{1}^{*} \psi_{2}^{*} .
\end{aligned}
$$

In both cases we could make one commutation because we had already proved Lemma $\mathrm{V}-4$ for this special situation and the other one because of Propositions $\mathrm{V}-2$ and $\mathrm{V}-3$. This proves Lemma $\mathrm{V}-4$.

We now have to consider the commutation relations of $\psi$ with itself.

$\mathbf{V}-5$. Theorem. Let $\mathcal{O}_{1}$ and $\mathcal{O}_{2}$ be two spacelike separated regions. Assume $\psi_{1} \in \mathfrak{F}\left(\mathcal{O}_{1}\right)$ and $\psi_{2} \in \mathfrak{F}\left(\mathcal{O}_{2}\right)$; then the following relations hold

$$
\begin{aligned}
& \psi_{1} \psi_{2}=\varepsilon \psi_{2} \psi_{1} \quad \text { with } \varepsilon=+1 \text { or }-1 . \\
& \psi_{1} \psi_{2}^{*}=\varepsilon \psi_{2}^{*} \psi_{1}
\end{aligned}
$$

$\varepsilon$ is independent of special choice of the $\psi$ 's and the two regions.

Proof. Let us consider besides $\mathcal{O}_{1}$ and $\mathcal{O}_{2}$ two other regions, $\mathcal{O}_{3}$ and $\mathcal{O}_{4}$, such that all four regions are spacelike separated from each other. Let $\psi_{3} \in \mathfrak{F}\left(\mathcal{O}_{3}\right)$ and $\psi_{4} \in \mathfrak{F}\left(\mathcal{O}_{4}\right)$. Then we get

$$
\begin{aligned}
\psi_{1} \psi_{2}^{*} & =\psi_{3} \psi_{3}^{*} \psi_{1} \psi_{2}^{*} \psi_{4} \psi_{4}^{*}=\psi_{3}\left(\psi_{3}^{*} \psi_{1}\right) \psi_{2}^{*}\left(\psi_{4} \psi_{4}^{*}\right) \\
& =\psi_{3} \psi_{2}^{*}\left(\psi_{3}^{*} \psi_{1}\right)\left(\psi_{4} \psi_{4}^{*}\right)=\left(\psi_{3} \psi_{2}^{*}\right) \psi_{3}^{*}\left(\psi_{1} \psi_{4}\right) \psi_{4}^{*} \\
& =\left(\psi_{3} \psi_{2}^{*}\right)\left(\psi_{1} \psi_{4}\right) \psi_{3}^{*} \psi_{4}^{*}=\psi_{3}\left(\psi_{2}^{*} \psi_{1}\right)\left(\psi_{4} \psi_{3}^{*} \psi_{4}^{*}\right) \\
& =\left(\psi_{2}^{*} \psi_{1}\right)\left(\psi_{3} \psi_{4} \psi_{3}^{*} \psi_{4}^{*}\right) .
\end{aligned}
$$

We multiply now both sides from the left with $\psi_{1}^{*} \psi_{2}$ and get

$$
\psi_{1}^{*} \psi_{2} \psi_{1} \psi_{2}^{*}=\psi_{3} \psi_{4} \psi_{3}^{*} \psi_{4}^{*} \text {. }
$$

From proposition $\mathrm{V}-2$ it follows that this expression is an element of the algebra $\mathfrak{A}$. From proposition $\mathrm{V}-3$ we find that the lefthand side commutes with all elements of $\mathfrak{A}$ which commute with $\mathfrak{A}\left(\mathcal{O}_{1} \cup \mathcal{O}_{2}\right)$; the righthand side commutes with all elements of $\mathfrak{A}$ which commute with $\mathfrak{A}\left(\mathcal{O}_{3} \cup \mathcal{O}_{4}\right)$. Choosing all possible situations for the four regions $\mathcal{O}_{i}$ we see that this expression commutes with all elements of $\mathfrak{A}$. Hence it is an element of the center of $\mathfrak{A}$. By assumption $\mathfrak{A}$ has only a trivial center. Hence we get

$$
\psi_{1}^{*} \psi_{2} \psi_{1}^{*} \psi_{2}=\psi_{1} \psi_{2} \psi_{1}^{*} \psi_{2}^{*}=\varepsilon
$$

Taking the adjoint of this equation we see $\varepsilon^{*}=\varepsilon$, and, since the $\psi^{\text {'s }}$ are unitary operators we find $\varepsilon=+1$ or -1 . This proves our theorem. 


\section{On the structure of the representation}

In this section, we want to inv sstigate the structure of the representation of the algebra $\mathfrak{B}$.

Before we can investigate the translations, we have to define them as automorphisms on the algebra $\mathfrak{B}$. To this end, we remark that we have representations $U_{0}(a)$ and $U_{1}(a)$ in the representation spaces $\mathfrak{S}_{0}$ resp. $\mathfrak{S}_{1}$. And, on the algebra $\mathfrak{A} \subset \mathfrak{B}$ we have already assigned to every translation an automorphism $\phi_{0}(a)$ mapping $\mathfrak{A}$ into $\mathfrak{A}$.

VI - 1. Definition. To every translation $a$ we define an automorphism of the algebra $\mathfrak{B}$ by the folowing transformation:

If $b$ is an element of $\mathfrak{A}$ we define

If $\psi=\psi(V)$ we define

$$
\phi(a) b=\phi_{0}(a) b .
$$

$$
\phi(a) \psi(V)=\psi\left(U_{0}(a) V U^{-1}(a)\right)
$$

and $\phi(a) \psi^{*}=(\phi(a) \psi)^{*}$.

With this definition we get:

VI - 2. Lemma. The positive functional $\omega$ on $\mathfrak{B}$ which is defined in Section IV is translation-invariant. This means for all $X \in \mathfrak{Z}$ we have

$$
\omega(\phi(a) X)=\omega(X) .
$$

Proof. This is an immediate consequence of the substitution rules which define the functional. Since $\omega$ is an invariant functional, the translations will be represented by unitary operators in the representation Hilbert space $\mathfrak{S}$ of $\omega$.

VI - 3. Definition. Let $\Omega$ be the cyclic vector defined by the representation of $\omega$. We define $\mathfrak{G}_{k}$ to be the smallest closed subspace containing all vectors of the form

$$
X \Omega, X \in \mathfrak{B} \quad \text { with } \quad N\left(\psi^{*}\right)-N(\psi)=k \quad k=0 \pm 1, \ldots
$$

VI - 4. Remarks. By definition of the functional $\omega$ we have that $\mathfrak{S}_{k}$ is orthogonal to $\mathfrak{G}_{i}$ for $i \neq k$ and $\mathfrak{G}$ is the direct sum over all $\mathfrak{G}_{k}$ :

$$
\mathfrak{G}=\sum_{-\infty}^{+\infty} \oplus \mathfrak{H}_{k} .
$$

By definition of $\mathfrak{G}_{k}$ we have also for $a \in \mathfrak{A}$,

$$
a \mathfrak{G}_{k} \subset \mathfrak{H}_{k} \text {. }
$$

This means every $\mathfrak{S}_{k}$ reduces the representation of the algebra $\mathfrak{A}$. We denote this subrepresentation by $D_{k}(\mathfrak{A})$.

In the same manner we see that the representation $U(X)$ of the translation group leaves each space $\mathfrak{S}_{k}$ invariant:

$$
U(X) \mathfrak{S}_{k}=\mathfrak{S}_{k} .
$$


From the fact that the $\psi$ 's are unitary operators and the definition of $\mathfrak{H}_{k}$, it follows immediately

$$
\begin{aligned}
\psi^{*} \mathfrak{G}_{k} & =\mathfrak{G}_{k+1} \\
\psi \mathfrak{S}_{k} & =\mathfrak{G}_{k-1} .
\end{aligned}
$$

VI - 5. Theorem. The weak closure of $D_{k}(\mathfrak{A})$ in $\mathfrak{G}_{k}$ coincides with $\mathfrak{L}\left(\mathfrak{G}_{k}\right)$, the space of all bounded linear operators in $\mathfrak{G}_{k}$.

Proof. Suppose $D_{k}(\mathfrak{U})$ is not irreducible; then there exists a vector $f$, $0 \neq f \in \mathfrak{S}_{k}$, such that $D_{k}(\mathfrak{U}) f$ is not dense in $\mathfrak{S}_{k}$. Let now $\mathfrak{M}$ be the orthogonal complement of $D_{k}(\mathfrak{Q}) f$. Let us denote by $\mathfrak{M}_{\psi}^{\prime}$ the subspace $(\psi)^{k} \mathfrak{M}$ in $\mathfrak{G}_{0}$. Since $\mathfrak{M}$ is invariant under the action of $D_{k}(\mathfrak{Q})$ we find

$$
\mathfrak{W}_{\psi_{1}}^{\prime}=\left(\psi_{1}\right)^{k} \mathfrak{W}=\left(\psi_{2}\right)^{k}\left(\psi_{2}^{*}\right)^{k}\left(\psi_{1}\right)^{k} \mathfrak{W}=\left(\psi_{2}\right)^{k} \mathfrak{W}^{\prime}=\mathfrak{W}_{\psi_{2}}^{\prime}=\mathfrak{W}^{\prime} .
$$

Here we have used that $\left(\psi_{2}^{*}\right)^{k}\left(\psi_{1}\right)^{k}$ is a unitary element in $\mathfrak{A}$ which therefore maps $\mathfrak{M}$ onto $\mathfrak{M}$.

The independence of the definition of $\mathfrak{M}^{\prime}$ from $\psi$ implies that $\mathfrak{M}^{\prime}$ is invariant under the action of $D_{0}(\mathfrak{A}(\mathcal{O})$ ) for all open bounded regions $\mathcal{O}$. Hence, $\mathfrak{W}^{\prime}$ is invariant under $D_{0}(\mathfrak{U})$. Since $D_{0}(\mathfrak{H})$ is irreducible we have $\mathfrak{W}^{\prime}$ is either $\mathfrak{H}_{0}$ or 0 . Therefore $\mathfrak{W}$ is either $\mathfrak{G}_{k}$ or 0 . But this contradicts the assumption that $D_{k}(\mathfrak{U})$ is reducible.

\section{On the spectrum of the translations}

In this section, we want to show that the unitary representation of the translation group fulfils the spectrum condition. We also want to compare the spectra of the translations in the spaces $\mathfrak{G}_{k}$ and $\mathfrak{G}_{-k}$.

We remark that all $\mathfrak{S}_{k}$ are invariant under the translations and we denote by $U_{k}(X)$ the restriction of $U(X)$ to $\mathfrak{G}_{k}$. We need a wellknown lemma (see [7], Lemma 9).

VII - 1. Lemma. If the spectrum of the representation $U_{k}(X)$ is contained in the closed forward lightcone $\overline{\mathscr{V}}+$, then the smallest convex closed cone containing this spectrum coincides with the forward light cone.

VII - 2. Lemma. The cluster decomposition property for the vacuum expectation value holds, provided the two clusters are neutral, i.e $N(\psi)-N\left(\psi^{*}\right)=0$ for both clusters.

Proof. Let $b$ be any element of the algebra $\mathfrak{Z}$; then we denote: $b(X)=U(X) b U^{-1}(X)$.

Let us consider matrix elements of the form

$$
\left(\Omega, b_{1} b_{2}(\mathbf{X}) b_{3} \ldots b_{4}(\mathbf{X}) \ldots b_{j} \Omega\right) .
$$

If all $b$ are products of elements of $\mathfrak{A}(\mathcal{O}), \mathfrak{F}(\mathcal{O}), \mathfrak{F}^{*}(\mathcal{O})$ for some open bounded region $\mathcal{O}$, then we have

$$
\lim _{\mathbf{X} \rightarrow \infty}\left(\Omega, b_{1} b_{2}(\mathbf{X}) b_{3} b_{4}(\mathbf{X}) \Omega\right)=\varepsilon \lim _{\mathbf{X} \rightarrow \infty}\left(\Omega, b_{1} b_{3} \ldots b_{2}(\mathbf{X}) b_{4}(\Omega) \ldots \Omega\right)
$$


where $\varepsilon$ is the number +1 or -1 . Let us denote by $P_{0}$ the projection onto the Hilbert space $\mathfrak{G}_{0}$. If now $b_{1} \cdot b_{3} \ldots$ and $b_{2} \cdot b_{4} \ldots$ are both neutral, i.e. elements of $\mathfrak{A}$, then we have

$\left(\Omega b_{1} \cdot b_{3} \cdot \ldots b_{2}(\mathbf{X}) b_{4}(\mathbf{X}) \ldots \Omega\right)=\left(\Omega, b_{1} \cdot b_{3} \ldots P_{0} b_{2}(\mathbf{X}) b_{4}(\mathbf{X}) \ldots \Omega\right)$

Since we know that in $\mathfrak{Y}_{0}$ the cluster decomposition property holds [9], we get:

$$
\lim _{\mathbf{X} \rightarrow \infty}\left(\Omega b_{1} b_{2}(\mathbf{X}) b_{3} b_{4}(\mathbf{X}) \ldots \Omega\right)=\varepsilon\left(\Omega, b_{1} b_{3} \ldots \Omega\right)\left(\Omega, b_{2} b_{4} \ldots \Omega\right)
$$

This result will also hold if we replace $b(X)$ by expressions $\int f(X-Y) \times$ $\times b(Y) d Y$ where $f$ is a strongly decreasing function.

VII - 3. Lemma [10]. Let us denote by $S_{k}$ the spectrum of the representation of $U_{k}(X)$ in $\mathfrak{G}_{k}$. Then we have the following additivity property

$$
S_{i}+S_{k} \subset S_{i+k} \quad i, k=0,1, \pm 2 \ldots
$$

Proof. Let $p$ be a point in the spectrum of $S_{i}$. Then, there exists a $\psi$ and a strongly decreasing function $f$ whose Fourier transform has support in a small neighbourhood of $p$ such that

$$
\int U(X)\left(\psi^{*}\right)^{i} U^{-1}(X) \quad f(X) d X \equiv \psi_{f}^{* i}
$$

does not annihilate the vacuum. In the same way, we find $\psi_{g}^{* k}$, where $\tilde{g}$ has support in a small neighbourhood of a point $q \in S_{k}$. Now

$$
\left(\Omega\left(\psi_{g}^{* k}\right)^{*}\left(\psi_{f}^{* i}\right)^{*}(\mathbf{X}) \psi_{f}^{* i}(\mathbf{X}) \psi_{g}^{* k} \Omega\right)
$$

converges for $\mathbf{X} \rightarrow \infty$ to

$$
\varepsilon\left(\Omega,\left(\psi_{g}^{* k}\right)^{*} \psi_{g}^{* k} \Omega\right)\left(\Omega,\left(\psi_{f}^{* i}\right)^{*} \psi_{f}^{* i} \Omega\right)
$$

which is not equal to zero. Hence

$$
\psi_{i}^{* i}(X) \psi_{g}^{* k} \Omega
$$

cannot be zero for all $X$. Hence in $S_{i+k}$ there exists a point in every neighbourhood of $p+q$. This then implies $p+q \in S_{i+k}$ q.e.d.

VII - 4. Theorem. The spectrum of $U(X)$ is contained in the forward light cone.

Proof. The proof will be by induction.

We know the representations $U_{1}(X)$ and $U_{0}(X)$ of the translations coincide with the two given representations which fulfil the spectrum condition by assumption. Now assume all representations $U_{i}(X)$ fulfil the spectrum-condition for $i=n, n-1, \ldots,-n+1$. We have to show that this is also true for $i=n+1$ and $-n$. From the additivity of the spectrum it follows that for $-n$

$$
S_{n}+S_{-n} \subset S_{0} .
$$

From this it follows that $S_{-n}$ is contained in a region $G$ which is bounded below in energy and which coincides asymptotically for high energies 
with the light cone. Assume now there is a point $p$ outside the forward light cone which is contained in $S_{-n}$; then $p+S_{0}$ must also be in $S_{-n}$ (since $S_{0}+S_{-n}\left(S_{-n}\right)$. But this is impossible because $p+S_{0}$ is not asymptotically contained in the light cone. Hence such points do not exist and we have $S_{-n} \subset \bar{V}+$.

By the same argument, with the relation

$$
S_{-n}+S_{n+1} \subset S_{1}
$$

we will find $S_{n+1} \subset \bar{V}^{+}$. This proves the theorem. We now want to study the spectra in greater detail.

VII - 5. Proposition. Let $f(z, p)$ be an entire function in $z \in \mathbf{C}^{4}$ with values in the set of Lorentz-invariant measures on Minkowski space. Let us define $S(f)$ to be the support of $f(p, p)$. Then $S(f)$ is a Lorentzvariant set.

Proof. By definition is $S(f)$ a closed set. Now let $q$ be a point in the complement of $S(f)$; then there exists a certain neighbourhood of $q$ which is in the complement of $S(f)$. Hence there exist certain subneighbourhoods $N_{1}, N_{2}$ such that $f(X, p)=0$ for $X \in N_{1}$ and $p \in N_{2}$. Since $N_{1}$ is open, we find by analytic combination $f(X, p)=0$ for all $X$ and $p \in N_{2}$. But $f(X, p)$ is Lorentz-invariant in $p$. Hence, $f(X, \Lambda p)=0$ for all $X$, all Lorentz transformations $\Lambda$ and $p \in N_{2}$. But this implies the complement of $S(f)$ is Lorentz invariant; hence $S(f)$ itself is Lorentz invariant q.e.d.

VII - 6. Proposition. The same assumptions as in VII -5 .

Let $f(z, p)=f_{s}(z, p)+f_{c}(z, p)$ be the splitting of $f(z, p)$ into its singular part and the part which is continuous with respect to theLebesgue measure. Then $f_{s}$ and $f_{c}$ are analytic functions in $z$ with values in the set of Lorentz-invariant measures.

Proof. The basis for this decomposition is the Lebesgue decomposition theorem (e.g. [11]). Thus, for every Lebesgue null-function $g$ we get that

$$
\int_{p} g(p) f(p, z)=\int_{p} g(p) f_{s}(p, z)
$$

is an entire holomorphic function in $z$. From this it follows in particular that if $E_{1} \subset E_{2}$ are two sets of Lebesgue measure zero and if for one $z$

$$
\int_{E_{1}}|f(p, z)|<\int_{E_{\mathrm{s}}}|f(p, z)|,
$$

then this is true for almost all $z$, since for any $g$ with support in $E_{2}-E_{1}$

$$
\int g(p) f(p, z) \neq 0 \quad \text { for one } z \text { implies }
$$

that this is true for almost all $z$. Hence

$$
\int_{E_{z}-E_{1}}|f(p, z)|>0 \quad \text { for almost all } z .
$$


Let $N$ be the set of Lebesgue zero sets. We have a semiordering and

$$
E_{1}<E_{2} \text { if } E_{1} \subset E_{2}
$$

$$
\int_{E_{1}}|f(p, z)|<\int_{E_{2}}|f(p, z)|
$$

for almost all $z$. Now the usual argument shows that there exists a maximal element $E_{0}$ in $N$ and one defines

$$
\int_{E} f_{s}(p, z)=\int_{E_{0} E} f(p, z) .
$$

Since $E_{0}$ is independent of $z$ we have that $f_{s}$ and $f_{c}=f-f_{s}$ fulfil all requirements of Prop. 6.

VII - 7. Theorem. Denote by $S_{k}$ the spectrum of the translation group in the Hilbert space $\mathfrak{S}_{k}$. Denote by $S_{k}^{0}$ the singular part with respect to the Lebesgue measure of $S_{k}$. Then we have

1) the supports of $S_{k}$, $S_{k}^{0}$ and $S_{k}-S_{k}^{0}$ are Lorentz invariant sets.

2) support $\quad S_{k}=$ support $S_{-k}$

$$
\text { support } \quad S_{k}^{0}=\operatorname{support} S_{-k}^{0}
$$

support $S_{k}-S_{k}^{0}=$ support $S_{-k}-S_{-k}^{0}$.

Physically speaking, this means the masses of particles and antiparticles are the same.

Proof. We remark first: let $\psi_{1}, \ldots, \psi_{K} \in \mathfrak{F}(0)$.

Then, the vectors of the form $\psi_{1}^{*} \psi_{2}^{*} \ldots \psi_{k}^{*} \Omega$ form a total set in $\mathfrak{S}_{k}$. This follows from the Reeh- Schlieder theorem.

Let us consider the commutator

$$
\begin{aligned}
& \left(\Omega, \psi_{1} \psi_{2} \ldots \psi_{n} U(X) \psi_{n+1}^{*} \ldots \psi_{2 n}^{*} \Omega\right)-\varepsilon^{n^{2}} \times \\
& \times\left(\Omega, \psi_{n+1}^{*} \ldots \psi_{2}^{*} U(-X) \psi_{1} \ldots \psi_{n} \Omega\right)=f(X)
\end{aligned}
$$

for $\psi_{i} \in \mathfrak{F}(\mathcal{O})$. Since $\mathcal{O}$ is bounded, this commutator vanishes in a region which is spacelike with respect to some double-cone $\left|X^{0}\right|-|\mathbf{X}|<M$. By the spectrum condition the Fourier transform of the first term has support in the forward light cone and the second has term support in the backward light cone. Hence by Dyson's representation [12] $f(X)$ is of the form

$$
f(X)=\int_{R|\geqq| U^{0}|\geqq U|} d U \varepsilon\left(X^{0}\right) \varphi\left((X-U)^{2}, U\right) .
$$

This implies for the Fourier transform

$$
f(p)=\varepsilon\left(p^{\circ}\right) \cdot \tilde{\varphi}\left(p^{2}, p\right),
$$

where $\tilde{\varphi}\left(p^{2}, z\right)$ is an entire analytic function in $z$. Now propositions VIII -5 and 6 give the desired result. 


\section{The case of many fields}

The introduction of many fields will be treated by an induction procedure. Assume we have introduced already $k$ "charged fields" $\psi^{1}, \ldots, \psi^{k}$. The algebra generated by the $k$ fields and by $\mathfrak{A}$ will be denoted by $\mathfrak{B}^{k}$ and its vacuum-representation by $D_{0}\left(\mathfrak{B}^{k}\right)$. Let now $D_{k+1}(\mathfrak{A}$ be the given representation of $\mathfrak{A}$. Then we have to extend this representation to a representation $D_{1}\left(\mathfrak{B}^{k}\right)$ of $\mathfrak{Z}^{k}$. To every operator $V^{k+1} \in F^{k+1}(\mathcal{O})$ which maps $D_{k+1}\left(\mathfrak{A} \cap \mathfrak{A}(\mathcal{O})^{\prime}\right)$ onto the corresponding ring in the vacuum-representation $D_{0}(\mathfrak{Q})$ of $\mathfrak{A}$ we construct an operator $W\left(V^{k+1}\right)$ which maps the representation space of $D_{1}\left(\mathfrak{B}^{k}\right)$ onto $D_{0}\left(\mathfrak{B}^{k}\right)$ such that $W^{-1}$ restricted to the vacuum sector coincides with $\left(V^{k+1}\right)^{-1}$ and such that $W\left(V_{1}^{k+1}\right) W^{-1}\left(V_{2}^{k+1}\right)$ is an element of the form $D_{0}(a)$ with $a \in \mathfrak{A}$. Having constructed the $W$ 's we can proceed as in Section IV and get a representation of the algebra $\mathfrak{B}^{k+1}$ generated by $\mathfrak{A}$ and $k+1$ charged fields.

VIII - 1. Proposition. Let $\mathfrak{Z}^{k}$ be an algebra containing $\mathfrak{A}$ and $k$ charged fields $\psi^{k}$ such that every neutral element of $\mathfrak{B}^{k}$ is contained in $\mathfrak{A}$.

Let $D_{k+1}(\mathfrak{Q})$ be a given representation of $\mathfrak{A}$ described in Section II; then there exists an extension of the representation $D_{k+1}$ of $\mathfrak{A}$ to a representation $D_{1}\left(\mathfrak{B}^{k}\right)$ of the algebra $\left(\mathfrak{Z}^{k}\right)$.

Proof. Denote by $\mathfrak{G}_{k+1}$ resp. $\mathfrak{G}_{0}$ the representation spaces of $D_{k+1}(\mathfrak{R})$ resp. $D_{0}(\mathfrak{U})$, and denote by $\phi_{k+1}$ the isomorphism

$$
D_{k+1}(\mathfrak{A}) \stackrel{\phi_{k+1}}{\longrightarrow} D_{0}(\mathfrak{U}) \text {. }
$$

Let $f \in \mathfrak{S}_{k+1}$; we define a positive linear functional on $\mathfrak{B}^{k}$ by

and

$$
\varrho(b)=0 \quad \text { if } b \text { is a non-neutral monomial of } \mathfrak{B}^{k}
$$

$$
\varrho(b)=\left(f, \phi_{k+1} D_{0}(b) f\right) \quad \text { if } b \text { is neutral and hence } b \in \mathfrak{A} \text {. }
$$

This functional is clearly linear und since the neutral part of every positive element in $\mathfrak{Z}^{k}$ is also positive, we see that $\varrho$ is a positive functional. If we denote the representation of $\varrho$ by $D_{1}\left(\mathfrak{B}^{k}\right)$ we see that $D_{1}\left(\mathfrak{B}^{k}\right)$ is an extension of $D_{k+1}(\mathfrak{Q})$ q.e.d.

If we restrict the representation $D_{0}\left(\mathfrak{B}^{k}\right)$ resp. $D_{1}\left(\mathfrak{Z}^{k}\right)$ to the algebra $\mathfrak{A}$, then these representations split into different subrepresentations which are characterized by a $k$-dimensional vector with integral components. We denote these Hilbert spaces by $\mathfrak{G}_{(0, \mathbf{a})}$ resp. $\mathfrak{G}_{(1, \mathbf{a})}$ and the corresponding representations by $D_{(0, \mathbf{a})}$ resp. $D_{(1, \mathbf{a})}$. What we have called $D_{0}$ becomes now $D_{(0,0)}$ and $D_{k+1}$ becomes $D_{(1,0)}$. To every $V \in F_{k+1}(\mathcal{O})$ we want to construct an operator $W(V)$ which maps the representation space of $D_{0}\left(\mathfrak{B}^{k}\right)$ onto the representation space of $D_{1}\left(\mathfrak{Z}^{k}\right)$.

VIII - 2. Definition. Let $V \in F_{k+1}(\mathcal{O})$ and $\mathcal{O}_{1}$ be spacelike with respect to $\mathcal{O}$. We define $W(V)$ as an operator mapping $\mathfrak{G}_{(0, \mathbf{a})}$ onto $\mathfrak{S}_{(1, \mathbf{a})}$. It 
must be of the form

$W(V) \mathfrak{S}_{(0, \mathbf{a})}=\alpha(\mathbf{a}) \tilde{\psi}_{1}^{* a_{1}} \tilde{\psi}_{2}^{* a_{\mathbf{2}}} \ldots \tilde{\psi}_{k}^{* a_{k}} V \psi_{k}^{a_{k}} \ldots \psi_{2}^{a_{\mathrm{s}}} \psi_{1}^{a_{1}} \mathfrak{G}^{(0, \mathbf{a})},|\alpha(\mathbf{a})|=\mathbf{1}$

With $\psi_{1} \in \mathfrak{F}^{1}(\mathcal{O}), \ldots, \psi_{k} \in \mathfrak{F}^{k}\left(\mathcal{O}_{1}\right)$, where $\tilde{\psi}$ denotes the representative of $\psi$ in the representation $D_{1}\left(\mathfrak{B}^{k}\right)$.

VIII - 3. Lemma. The operators $W(V)$ are unitary.

Furthermore, their definition is independent of the special choice of $\psi_{s}^{\prime}$. For $V_{1}, V_{2} \in F^{k+1}(\mathcal{O})$ we get

resp.

$$
W^{-1}\left(V_{1}\right) W\left(V_{2}\right) \in\left(D_{0} \mathfrak{A}(\mathcal{O})\right)
$$

$$
W\left(V_{1}\right) W^{-1}\left(V_{2}\right) \in D_{1}(\mathfrak{A}(\mathcal{O})) .
$$

Proof. The unitarity of $W(V)$ follows immediately from the unitarity of $V$ and the unitarity of the $\psi_{s}^{\prime}$. We show now the independence of $W(V)$ from the special choice of the $\psi_{s}^{\prime}$. Let $\psi_{j}, \psi_{j}^{\prime} \in \mathfrak{F}^{j}\left(\mathcal{O}_{1}\right)$; then there exists a unitary element $U$ in $\mathfrak{A}\left(\mathcal{O}_{1}\right)$ such that

Hence we get

$$
\psi_{k}^{a_{k}} \ldots \psi_{1}^{a_{1}}=D_{0}(U) \psi_{a}^{a_{k}} \ldots \psi_{1}^{\prime a_{1}}
$$

$$
\begin{aligned}
\alpha(\mathbf{a}) \tilde{\psi}_{1}^{* a_{1}} & \ldots \tilde{\psi}_{k}^{* a_{k}} V \psi_{k}^{a_{k}} \ldots \psi_{1}^{a_{1}} \mathfrak{G}_{(0, \mathbf{a})} \\
& =\alpha(\mathbf{a}) \tilde{\psi}_{1}^{\prime * a_{1}} \ldots \tilde{\psi}_{k}^{\prime a_{k}} D_{1}\left(U^{*}\right) V D_{0}(U) \psi_{k}^{\prime} a_{k} \ldots \psi_{1}^{\prime a_{1}} \mathfrak{G}_{(0, \mathbf{a})} \\
& =\alpha(\mathbf{a}) \tilde{\psi}_{1}^{\prime * a_{1}} \ldots \tilde{\psi}_{k}^{\prime * a_{k}} D_{(1, \mathbf{0})}\left(U^{*}\right) V D_{(0, \mathbf{0})}(U) \psi_{k}^{\prime a_{k}} \ldots \psi_{1}^{\prime a_{1}} \mathfrak{G}_{(0, \mathbf{a})} \\
& =\alpha(\mathbf{a}) \tilde{\psi}_{1}^{* a_{1}} \ldots \tilde{\psi}_{k}^{* a_{k}} V{\psi_{k}^{\prime}}^{a_{k}} \ldots \psi_{1}^{\prime a_{1}} \mathfrak{G}_{(0, \mathbf{a})} .
\end{aligned}
$$

Here we have used that $\mathcal{O}$ and $\mathcal{O}_{1}$ are spacelike separated and consequently the relation

holds.

$$
V D_{(0,0)}(U)=D_{(1,0)}(U) V
$$

The relations

and

$$
W^{-1}\left(V_{1}\right) W\left(V_{2}\right) \in D_{0}(\mathfrak{U}(\mathcal{O}))
$$

$$
W\left(V_{1}\right) W^{-1}\left(V_{2}\right) \in D_{1}(\mathfrak{A}(\mathcal{O}))
$$

are an immediate consequence of Lemma III - 3 q.e.d.

VIII - 4. Lemma. Let $\mathcal{O}$ and $\mathcal{O}_{1}$ be two spacelike separated regions, $V \in F^{k+1}(\mathcal{O}), a \in \mathfrak{A}\left(\mathcal{O}_{1}\right)$; then we have the relation

$$
W(V) D_{0}(\mathbf{a})=D_{1}(\mathbf{a}) W(V)
$$

Furthermore, the numbers $\alpha($ a) can be adjusted in such a way that we have for $\psi_{k} \in \mathfrak{F}^{k}\left(\mathcal{O}_{1}\right)$ the relations

$$
W(V) \psi_{k}=\mu_{k} \tilde{\psi}_{k} W(V), \quad \mu_{k}=1 \text { or }-1 .
$$


Proof. Let $\mathcal{O}_{2}$ be a region spacelike separated from $\mathcal{O}_{\text {and }} \mathcal{O}_{1}$, and $\psi_{j} \in \mathfrak{F}^{j}\left(\mathcal{O}_{2}\right)$; then we have with the notation $\psi^{\mathbf{x}}=\psi_{k}^{x_{k}} \ldots \psi_{k_{1}}^{x_{1}}$ :

This is equivalent to

$$
\begin{aligned}
W(V) D_{(0, \mathbf{x})}(a) & =\alpha(\mathbf{x}) \tilde{\psi}^{-\mathbf{x}} V \psi^{\mathbf{x}} D_{(0, \mathbf{x})}(a) \\
& =\alpha(\mathbf{x}) \tilde{\psi}^{-\mathbf{x}} V D_{(0,0)}(a) \psi^{\mathbf{x}} \\
& =\alpha(\mathbf{x}) \tilde{\psi}^{-\mathbf{x}} D_{(1, \mathbf{0})}(a) V \psi^{\mathbf{x}} \\
& =D_{(1, \mathbf{x})}(a) \alpha(\mathbf{x}) \tilde{\psi}^{-\mathbf{x}} V \psi^{\mathbf{x}} .
\end{aligned}
$$

$$
W(V) D_{0}(\mathbf{a})=D_{1}(\mathbf{a}) W(V) .
$$

Now we consider the expression

$$
W^{-1}(V) \tilde{\psi}_{k}^{*} W(V) \psi_{k}
$$

for a situation where $V$ and $\psi_{k}$ belong to spacelike separated regions. Because of Lemma VIII - 3 this expression is independent of the special choice of $V$ and $\psi$ :

$$
\begin{aligned}
W^{-1}\left(V^{\prime}\right) \tilde{\psi}_{k}^{\prime *} W\left(V^{\prime}\right) \psi_{k}^{\prime} & =W^{-1}\left(V^{\prime}\right) \tilde{\psi}_{k}^{*} W\left(V^{\prime}\right) D_{0}\left(U_{1}\right) \psi_{k} \\
& =W^{-1}\left(V^{\prime}\right) \tilde{\psi}_{k}^{*} W\left(V^{\prime}\right) \psi_{k} \\
& =W^{-1}\left(V^{\prime}\right) \tilde{\psi}_{k}^{*} D_{1}\left(U_{2}\right) W(V) \psi_{k} \\
& =W^{-1}(V) \tilde{\psi}_{k}^{*} W(V) \psi_{k} .
\end{aligned}
$$

This implies that this expression commutes with $D_{0}(\mathfrak{A})$ and is hence in each sector $\mathfrak{G}_{(0, \mathbf{a})}$ equal to a constant $\beta_{k}(\mathbf{a})$. We get

$$
\begin{aligned}
\beta_{k}(\mathbf{a}) & =\left(\psi^{-\mathbf{a}} \Omega, W^{-1}(V) \tilde{\psi}_{k}^{*} W(V) \psi_{k} \psi^{-\mathbf{a}} \Omega\right) \\
& =\left(W(V) \psi^{-\mathbf{a}} \Omega, \tilde{\psi}_{k}^{*} W(V) \psi_{k} \psi^{-\mathbf{a}} \Omega\right) \\
& =\bar{\alpha}(\mathbf{a}) \alpha\left(\mathbf{a}-\mathbf{1}_{k}\right)\left(\tilde{\psi}^{-\mathbf{a}} V \Omega \tilde{\psi}_{k}^{*} \tilde{\psi}_{k} \tilde{\psi}^{-\mathbf{a}} V \Omega\right)=\bar{\alpha}(\mathbf{a}) \alpha\left(\mathbf{a}-\mathbf{1}_{k}\right) .
\end{aligned}
$$

Putting now $\alpha(\mathbf{a})=\mu_{1}^{a_{1}} \cdot \mu_{2}^{a_{2}} \ldots \mu_{k}^{a_{k}}$ we get

$$
\beta_{k}(\mathfrak{a})=\mu_{k}, \quad \text { q.e.d. }
$$

Choosing now $\mu_{k}=1$ we are back to the original situation of introducing one field. This closes the induction procedure.

We have now introduced $n$ different fields which commute with one another. Making finally a Klein transformation (e.g. [13]) we get the result.

VIII - 5. Theorem. Assume there are $n$ different representations of a local ringsystem fulfilling all assumptions of Section II. Then we can introduce $n$ charged fields $\psi_{i}, i=1 \ldots n$, such that

1) Each $\psi_{i}$ either commutes or anti-commutes for spacelike separations.

2) Between the different fields we have the normal commutation relations.

3) The given representations appear as representations in superselection sectors. 


\section{On the uniqueness of the imbedding procedure}

This section is devoted to the uniqueness problem of the imbeddingprocedure. In particular, we want to show that we can replace the given representations $D_{k}, k=0,1, \ldots, n$, by unitarily equivalent representations. Furthermore, we want to show that the result of our construction is independent of the order of succession in the construction procedure.

If we have two unitarily equivalent representations $D_{1}$ and $D_{2}$ of the algebra $\mathfrak{A}$, we write

$$
D_{1} \cong D_{2} .
$$

If we have a field theory containing $n$ fields $\psi_{i}$, we denote the different super-selection sectors by a vector a whose components are integral. In particular $\mathfrak{G}_{0}$ is the vacuum sector and

$$
\mathfrak{G}_{\mathbf{a}}=\psi_{1}^{* a_{1}} \psi_{2}^{* a_{2}} \ldots \psi_{n}^{* a_{n}} \mathfrak{G}_{\mathbf{0}} \equiv \psi^{* \mathbf{a}} \mathfrak{G}_{\mathbf{0}} .
$$

By $D_{\mathfrak{a}}$ we denote the representation of the original algebra in the sector $\mathfrak{G}_{\mathfrak{a}}$.

IX - 1. Lemma. Let us take a representation $D_{\mathfrak{a}}, \mathfrak{a} \neq \mathbf{0}$, and the representation $D_{0}$, and let us construct a field $\phi$ as described in Section IV connecting the representations $D_{0}$ and $D_{\mathbf{a}}$. Then we get a string of representations $D_{i}^{\prime}$ such that

$$
D_{i}^{\prime} \cong D_{i \mathrm{a}} .
$$

Proof. Let us denote by $F(\mathcal{O})$ the set of all $V$, mapping $\left(D_{1}^{\prime}(\mathcal{O})\right)^{\prime} \cap D_{1}^{\prime}(\mathfrak{R})$ onto $\left(D_{0}^{\prime}(\mathcal{O})\right)^{\prime} \cap D_{0}^{\prime}(\mathfrak{R})$.

Since $D_{1}^{\prime}=D_{\mathrm{a}}$ and $D_{0}^{\prime}=D_{0}$ we find that

$$
P_{0} \psi^{\mathbf{a}} P_{\mathbf{a}} \text {, for } \psi_{i} \in \mathfrak{F}_{i}(\mathcal{O})
$$

and $P_{\mathbf{b}}$ the projection onto $\mathfrak{G}_{\mathbf{b}}$, is an element of $F(\mathcal{O})$. Hence

$$
F(\mathcal{O})=\left\{P_{\mathbf{0}} \psi^{\mathrm{a}} P_{\mathrm{a}} D_{\mathrm{a}}(U) ; U \in \mathfrak{A}(\mathcal{O}), \quad U \text { unitary }\right\} .
$$

This implies that the vacuum functional $\omega$ on the algebra $\mathfrak{B}=\{\mathfrak{A}, F(\mathcal{O})\}$ coincides with the vacuum expectation values of the subalgebra $\mathfrak{B}^{\mathfrak{a}}$ of $\mathfrak{B}^{n}$ which is the subalgebra generated by $\mathfrak{A}$ and all fields of the form $\psi^{\mathrm{a}} . \mathfrak{B}$ and $\mathfrak{B}^{\mathrm{a}}$ are in a natural manner isomorphic algebras. The cyclic subspace of the algebra $\mathfrak{B}^{\mathrm{a}}$ is $\sum_{n=-\infty}^{+\infty} \mathfrak{F}_{n \mathbf{a}}$. Hence the representation of $\mathfrak{B}^{\mathrm{a}}$ restricted to this subspace is equivalent to the representation of $\mathfrak{B}$, i.e.

$$
D(\mathfrak{B}) \cong D\left(\mathfrak{B}^{\mathrm{a}}\right) \text {. }
$$

Restricting to the representation of $\mathfrak{A}$ we get the desired result.

IX - 2. Lemma. Assume that we have two pairs of representations $D_{1}, D_{0}$ and $D_{1}^{\prime}, D_{0}^{\prime}$ such that $D_{1} \cong D_{1}^{\prime}, D_{0} \cong D_{0}^{\prime}$. 
Let us construct the fields $\psi$ resp. $\psi^{\prime}$ connecting the representations $D_{0}$ and $D_{1}$ resp. $D_{0}^{\prime}$ and $D_{1}^{\prime}$. Then we get two strings of representations $D_{i}$ resp. $D_{i}^{\prime}$ for $\mathfrak{A}$ such that $D_{i} \cong D_{i}^{\prime}$.

Moreover, if we denote by $\mathfrak{B}$ resp. $\mathfrak{B}^{\prime}$ the algebras generated by $\{\mathfrak{A}$ and $F(\mathcal{O})\}$ resp. $\left\{\mathfrak{A}\right.$ and $\left.F^{\prime}(\mathcal{O})\right\}$, then we have a natural isomorphism between $\mathfrak{B}$ and $\mathfrak{B}^{\prime}, \mathfrak{B}=\phi\left(\mathfrak{B}^{\prime}\right)$, such that

$$
D(\mathfrak{B}) \cong D^{\prime}\left(\phi^{-1}(\mathfrak{B})\right) \text {. }
$$

Proof. Let $U_{0}$ resp. $U_{1}$ be the unitary operators

$$
U_{0} D_{0} U_{0}^{-1}=D_{0}^{\prime} \quad \text { resp. } \quad U_{1} D_{1} U_{1}^{-1}=D_{1}^{\prime} .
$$

Let us denote be $F(\mathcal{O})$ resp. $F^{\prime}(\mathcal{O})$ the family of operators $V$ resp. $V^{\prime}$ establishing the local unitary equivalence. For $V^{\prime} \in F^{\prime}(\mathcal{O})$ we get clearly

or in general

$$
U_{0}^{-1} V^{\prime} U_{1} \in F(\mathcal{O})
$$

$$
U_{0}^{-1} F^{\prime}(\mathcal{O}) U_{1}=F(\mathcal{O}) \text {. }
$$

This establishes in a natural manner an isomorphism between the two algebras $\mathfrak{B}$ and $\mathfrak{B}^{\prime}$.

Let us denote by $\psi$ resp. $\psi^{\prime}$ the two fields and let $M$ be a neutral monomial $M=M\left(a_{i}, \psi\left(V_{i}\right)\right)$.

We want to show that the relation

$$
U_{0} P_{0} M P_{0} U_{0}^{-1}=P_{0}^{\prime} M^{\prime} P_{0}^{\prime}
$$

holds. We do this by induction with respect to the number $N(\psi)$ of $\psi_{s}^{\prime}$ which are in $M$.

We have the three possibilities

$$
M_{n+1}=\left\{\begin{array}{l}
M_{k} M_{n+1-k} \\
a_{1} \psi\left(V_{2}\right) M_{n} \psi^{*}\left(V_{3}\right) a_{4} \\
a_{1} \psi^{*}\left(V_{2}\right) M_{n} \psi\left(V_{3}\right) a_{4} .
\end{array} \quad 1 \leqq k \leqq n\right.
$$

The first case is trivial. Now the second:

The third case:

$$
\begin{aligned}
U_{0} P_{0} M_{n+1} P_{0} U_{0}^{-1} & =U_{0} D_{0}\left(a_{1}\right) V_{2} D_{1}\left(M_{n}\right) V_{3}^{-1} D_{0}\left(a_{n}\right) U_{0}^{-1} \\
& =D_{0}^{\prime}\left(a_{1}\right) U_{0} V_{2} U_{1}^{-1} D_{1}^{\prime}\left(M_{n}\right) V_{1} V_{3}^{-1} U_{0}^{-1} D_{1}\left(a_{4}\right) \\
& =P_{0}^{\prime} M_{n+1}^{\prime} P_{0}^{\prime} .
\end{aligned}
$$

$$
\begin{aligned}
U_{0} P_{0} M_{n+1} p_{0} U_{0}^{-1} & =U_{0} D_{0}\left(a_{1}\right) P_{0} \psi^{*}\left(V_{2}\right) M_{n} \psi\left(V_{3}\right) P_{0} D_{0}\left(a_{4}\right) U_{0}^{-1} \\
& =D_{0}^{\prime}\left(a_{1}\right) U_{0} P_{0} \psi^{*}\left(V_{2}\right) M_{n} \psi\left(V_{3}\right) P_{0} U_{0}^{-1} D_{0}^{\prime}\left(a_{4}\right) \\
& =D_{0}^{\prime}\left(a_{1}\right) U_{0} \phi\left(V_{2}^{-1} D_{0}\left(M_{n}\right) V_{3}\right) U_{0}^{-1} D_{0}^{\prime}\left(a_{4}\right) \\
& =D_{0}^{\prime}\left(a_{1}\right) U_{0} \phi\left\{U_{1}^{-1}\left(U_{1} V_{2}^{-1} U_{0}^{-1} D_{0}^{\prime}\left(M_{n}\right) U_{0} V_{3} U_{1}^{-1}\right) U_{1}\right\} \\
& =D_{0}^{\prime}\left(a_{1}\right) U_{0} \phi\left\{U_{1}^{-1} D_{1}^{\prime}\left(\psi^{\prime}\left(V_{2}\right) M_{n}^{\prime} \psi\left(V_{3}\right)\right) U_{1}\right\} U_{0}^{-1} D_{0}^{\prime}\left(a_{4}\right) .
\end{aligned}
$$

Here $\phi$ denotes the isomorphism $D_{1} \rightarrow D_{0}$. 
We have

$$
D_{0}^{\prime}=U_{0} D_{0} U_{0}^{-1}=U_{0} \phi\left(D_{1}\right) U_{0}^{-1}=U_{0} \phi\left(U_{1}^{-1} D_{1}^{\prime} U_{1}\right) U_{0}^{-1} .
$$

Hence we get

$$
\begin{aligned}
U_{0} P_{0} M_{n+1} P_{0} U_{0}^{-1} & =D_{0}^{\prime}\left(a_{1}\right) D_{0}^{\prime}\left(\psi^{\prime *}\left(V_{2}\right) M_{n}^{\prime} \psi\left(V_{3}\right)\right) D_{0}^{\prime}\left(a_{4}\right) \\
& =P_{0}^{\prime} M_{n+1}^{\prime} P_{0}^{\prime} .
\end{aligned}
$$

Since $U_{0}$ maps the vacuum onto the vacuum, we see that the vacuum expectation values coincide. Hence we get the desired equivalences.

IX - 3. Corollary. Assume we have given $n+1$ representations $D_{i}(\mathfrak{A}), i=0,1, \ldots, n$, fulfilling the assumptions of Section II. $D_{0}(\mathfrak{A})$ is the vacuum representation. Let us construct the $n$ charged fields $\psi_{i}$; then the commutation relation of the field $\psi_{i}$ dependes only on the class of representations equivalent to $D_{i}(\mathfrak{A})$.

This is a trivial consequence of the two preceding lemmas.

IX - 4. Theorem. Assume we have $n+1$ pairs of representations $D_{i}, D_{i}^{\prime}, i=0,1, \ldots, n$, such that $D_{i} \cong D_{i}^{\prime}$. Let us construct the fields $\psi_{i}$ resp. $\psi_{i}^{\prime}$ connecting all these representations. Then we have a natural isomorphism $\phi\left(\mathfrak{Z}^{\prime n}\right)=\mathfrak{Z}^{n}$ between the two algebras $\mathfrak{Z}^{n}$ and $\mathfrak{B}^{n^{\prime}}$ such that

$$
D\left(\mathfrak{Z}^{n}\right) \cong D^{\prime}\left(\phi^{-1}\left(\mathfrak{B}^{n^{\prime}}\right)\right) .
$$

In particular we get for $\mathfrak{A}$ two sets of representations $D_{\mathrm{a}}$ and $D_{\mathbf{a}}^{\prime}$ such that

$$
D_{\mathbf{a}} \cong D_{\mathbf{a}}^{\prime}
$$

Proof. By induction: assume we have established the equivalence for the algebra $\mathfrak{Z}^{k}$ and we have found the isomorphism $\phi_{k}\left(\mathfrak{Z}^{k}\right)=\mathfrak{Z}^{k}$. Then we have

$$
D_{0}\left(\mathfrak{Z}^{k}\right) \cong D_{0}^{\prime}\left(\phi_{k}^{-1}(\mathfrak{B})\right)
$$

This means we have a unitary operator $U_{k}$ such that

$$
U_{k} D_{0}\left(\mathfrak{Z}^{k}\right) U_{k}^{-1}=D_{0}^{\prime}\left(\phi_{k}^{-1}(\mathfrak{Z})\right) .
$$

Let us denote by $U_{k+1}$ the operator

$$
U_{k+1} D_{k+1}(\mathfrak{A}) U_{k+1}^{-1}=D_{k+1}^{\prime}(\mathfrak{A})
$$

and by $\phi_{k+1}$ resp. $\phi_{k+1}^{\prime}$ the isomorphismus $D_{k+1} \stackrel{\phi_{k+1}}{\longrightarrow} D_{0}$ resp. $D_{k+1}^{\prime} \stackrel{\phi_{k+1}^{\prime}}{\longrightarrow} D_{0}^{\prime}$.

We construct now, according to Proposition VIII - 1, a positive functional on $\mathfrak{B}^{k}$ by

$\varrho(b)=0$ if $b$ is a non-neutral monomial of $\mathfrak{Z}^{k}$ and

$\varrho(b)=\left(f, \phi_{k+1}^{-1} D_{0}(b) f\right)$ if $b$ is a neutral element of $\mathfrak{Z}^{k}$. 
Here $f \in \mathfrak{S}_{k+1}$, the representation space of $D_{k+1}(\mathfrak{A})$. For neutral elements, we have the following equations

$$
\begin{aligned}
\varrho(b) & =\left(f, D_{k+1}(b) f\right)=\left(f, U_{k+1}^{-1} D_{k+1}^{\prime}(b) U_{k+1} f\right) \\
& =\left(U_{k+1} f, \phi_{k+1}^{\prime-1}\left(D_{0}^{\prime}(b)\right) U_{k+1} f\right) .
\end{aligned}
$$

$b$ is a neutral element and hence an element of $\mathfrak{A}$. This means the element $b \in \mathfrak{Z}^{k}$ has to be identified with the element $\phi_{k}^{-1}(b) \in \mathfrak{Z}^{\prime k}$.

$$
\varrho(b)=\left(U_{k+1} f, \phi_{k+1}^{\prime-1}\left(D_{0}\left(\phi^{-1} b\right)\right) U_{k+1} f\right)=\varrho^{\prime}\left(\phi_{k}^{-1} b\right)
$$

Hence the representations

$$
D_{1}(\mathfrak{Z}) \text { and } D_{1}^{\prime}\left(\phi^{-1}(\mathfrak{B})\right)
$$

are unitarily equivalent:

$$
D_{1}(\mathfrak{Z}) \cong D_{1}^{\prime}\left(\phi^{-1}(\mathfrak{Z})\right)
$$

Applying Lemma IX -2 , we get the desired result.

IX - 5. Lemma. Assume we have a field-theory containing $n$ fields; then we have a set of representations for $\mathfrak{A}$ labeled by an $n$-vector a with integral components.

Let us pick $k$ different representations

$$
D_{\mathrm{a}_{i}} \quad i=1,2, \ldots, k
$$

and the vacuum representation $D_{\mathbf{0}}$. Let us construct with these representations a field-theory containing $k$ fields. Then we get for the algebra $\mathfrak{A}$ a set of representations $D_{\mathbf{k}}^{\prime}$ labeled by a $k$-vector $\mathbf{b}$ such that

$$
D_{\mathbf{b}}^{\prime} \cong D_{b_{1} \mathbf{a}_{1}+b \cdot \mathbf{a}_{2} \cdots+b_{k} \mathbf{a}_{k}} .
$$

Proof. Let us denote the subalgebra of $\mathfrak{B}^{n}$ which is generated by $\mathfrak{A}$ and the fields $\psi^{\mathrm{a}_{i}}, i=1 \ldots k$, by $\mathfrak{Z}^{k}$. Then $\mathfrak{B}^{k}$ applied to the vacuum generates the Hilbert space $\mathfrak{H}=\sum_{n_{1} \cdots n_{k}} \mathfrak{Y}_{n_{1} \mathbf{a}_{1}}+n_{2} \mathbf{a}_{2}+\cdots n_{k} \mathbf{a}_{k}$. Let us denote by $\mathfrak{B}^{j}$ the algebra generated by $\mathfrak{A}$ to gether with the first $j$ fields, by $\mathfrak{S}_{j}$ the Hilbert space $\mathfrak{H}_{j}=\sum_{n_{1} \cdots n_{j}} \mathfrak{S}_{n_{1} \mathbf{a}_{1}+\cdots n_{j} \mathbf{a}_{j}}$ and by $\mathfrak{G}_{j, 1}$ the Hilbert space $\mathfrak{S}_{j, 1}=\sum_{n_{1} \cdots n_{j}} \mathfrak{S}_{n_{1} \mathbf{a}_{1}+\cdots+n_{j} \mathbf{a}_{j}+\mathbf{a}_{j+1}} \cdot \mathfrak{G}_{j}$ and $\mathfrak{S}_{j, 1}$ are both Hilbert spaces which are invariant under the algebra $\mathfrak{B}^{j}$. The two representations of $\mathfrak{B}^{j}$ are clearly locally unitarily equivalent. This equivalence is established by the operators

$$
E_{j} D(U) \psi^{\mathbf{a}_{j+1}} E_{j, 1}=W \quad \text { where } \quad \psi_{i} \in \mathfrak{F}_{i}(\mathcal{O}), \quad U \in \mathfrak{U}(\mathcal{O})
$$

and $E_{j}$ resp. $E_{j, 1}$ the projections onto $\mathfrak{G}_{j}$ resp. $\mathfrak{S}_{j, 1}$. Hence, by Lemma IX -1 , the theory constructed by the two representations of $\mathfrak{B}^{i}$ in $\mathfrak{S}_{j}$ resp. $\mathfrak{S}_{j, 1}$ and the $W_{s}^{\prime}$ will be unitarily equivalent to the representation of the algebra $\mathfrak{B}^{j+1}$ in the Hilbert space $\mathfrak{G}_{j+1}$. 
Now, the Hilbert space $\mathfrak{S}_{\mathbf{a}_{j+1}}$ is clearly a cyclic subspace of the algebra $\mathfrak{B}^{j}$ in $\mathfrak{G}_{j, 1}$. Hence for every $f$ in $\mathfrak{G}_{\mathfrak{a}_{j, 1}}$ and every neutral element $b \in \mathfrak{B}^{j}$ we get

$$
\left(f, D_{1}(b) f\right)=\left(f, D_{\mathbf{a}_{j+1}}(b) f\right)=\left(f, \phi_{j+1}^{-1}\left(D_{0}(b)\right) f\right) .
$$

Here $D_{1}\left(\mathfrak{B}^{j}\right)$ denotes the representation of $\mathfrak{Q}^{j}$ in $\mathfrak{S}_{j, 1}$, and $\phi_{j+1}$ the isomorphism

$$
\phi_{j+1}\left(D_{\mathrm{a}_{j+1}}(\mathfrak{R})\right)=D_{0}(\mathfrak{R}) .
$$

This means the representation $D_{1}(\mathfrak{B})$ is uniquely defined by the representation of $\mathfrak{B}$ in $\mathfrak{G}_{j}$ and the representation $D_{\mathbf{a}_{j+1}}$ of $\mathfrak{A}$. From this follows our lemma by induction.

We collect all the results of this section and get:

IX - 6. Theorem. If $S$ is a given finite set of locally unitarily equivalent representations of $\mathfrak{A}$ containing the vacuum representation, then the imbedding procedure is uniquely defined up to unitary equivalence by the equivalence-classes of the given representations, and is also independent of the numbering of the elements in $S$.

\section{Degeneracies and their reduction}

Starting from $n+1$ representations of the algebra $\mathfrak{A}$ we have constructed a field theory containing $n$ fields $\psi_{i}$. As a consequence of this, we get a family of representations for $\mathfrak{A}$ which are labeled by a vector $\mathfrak{a}$ with integral component. There is no reason to believe that in the general case all representations of $\mathfrak{A}$ we get by this construction must be different. In this section, we want to study what happens if two of these representations are equivalent. In particular, we want to show that in such a situation our scheme can be reduced in such a way that all representations are different. We first investigate the degeneracies.

The first result is a simple consequence of Lemmas IX - 1, 2 .

$\mathbf{X}$ - 1. Proposition. From $D_{\mathbf{a}} \cong D_{\mathbf{b}}$ follows

$$
D_{n \mathbf{a}} \cong D_{n \mathbf{b}} \quad n=0, \pm 1, \pm 2, \ldots
$$

$\mathbf{X}$ - 2. Lemma. From $D_{\mathbf{a}} \cong D_{\mathbf{b}}$ follows

and in particular

$$
D_{n \mathbf{a}+m \mathbf{b}} \cong D_{(n+m) \mathbf{a}} \cong D_{(n+m) \mathbf{b}},
$$

$$
D_{\mathbf{a}-\mathbf{b}} \cong D_{\mathbf{0}} \text {. }
$$

Proof. Nowhere in our construction of charged fields did we use the fact that the given representations of $\mathfrak{A}$ are not equivalent. Hence we can apply all results which we have obtained so far to our present situation. Assume we have two fields such that

$$
D_{0,1} \cong D_{1,0} ;
$$


then there exist unitary operators $U_{n}$ such that

$$
U_{n} D_{0, n} U_{n}^{-1}=D_{n, 0} \text {. }
$$

Let $f \in \mathfrak{G}_{0, n}$ and $\mathfrak{B}^{1}$ be the algebra genrated by $\mathfrak{U}$ and $\psi_{1}$; then we get for every neutral element in $\mathfrak{\mathfrak { Z }}_{1}$

$$
(f, D(b) f)=\left(f, D_{0, n}(b) f\right)=\left(U_{n} f, D_{n, 0}(b) U_{n} f\right) \text {. }
$$

But the vector $U_{n} f \in \mathfrak{S}_{n, 0}$. Hence, we get

$$
D_{m, n} \cong D_{n+m, 0} \text {. }
$$

Assume now $D_{1,0} \cong D_{\mathrm{a}}$ and $D_{0,1} \cong D_{\mathrm{b}}$; then we get the desired result.

$\mathbf{X}$ - 3. Proposition. Suppose we have the two representations $D_{0}, D_{1}$ of $\mathfrak{A}$. Assume moreover $D_{0} \cong D_{1}$; then $D_{n} \cong D_{0}$ for all $n$.

Proof. If we interchange the role of $D_{0}$ and $D_{1}$, we get

$$
D_{n} \cong D_{1-n} \text {. }
$$

Applying Proposition $X-1$ to the pairs $\left(D_{k}, D_{0}\right)$ and $\left(D_{k}, D_{1}\right)$ we find:

$$
D_{n k} \cong D_{1+n(k-1)} \text {. }
$$

Combining both results, we get:

$$
D_{n k} \cong D_{-n(k-1)} \text {. }
$$

The application of Lemma $\mathrm{X}-2$ gives:

$$
D_{n(2 k-1)} \cong D_{0} \text {. }
$$

But $n(2 k-1)$ runs through all the integers q.e.d.

$\mathbf{X}$ - 4. Lemma. From $D_{\mathbf{a}} \cong D_{0}$ follows

$$
D_{\mathrm{b}} \cong D_{\mathrm{b}+n \mathrm{a}} \text { for all } \mathrm{b} \text { and all } n=0, \pm 1, \pm 2, \ldots
$$

Proof. If we have a field theory with two fields such that

$$
D_{0,1} \cong D_{0,0} \text {, }
$$

then there exist unitary operators $U_{n}$ such that

$$
U_{n} D_{0, n} U_{n}^{-1}=D_{0,0} \text {. }
$$

Let $f \in \mathfrak{S}_{0},{ }_{n}$ and $\mathfrak{B}^{1}$ be the algebra generated by $\mathfrak{A}$ and $\psi_{1}$; then we get for every neutral element in $\mathfrak{Z}_{1}$

$$
(f, D(b) f)=\left(f, D_{0, n}(b) f\right)=\left(U_{n} f, D_{0,0}(b) U_{n} f\right) .
$$

The vector $U_{n} f \in \mathfrak{H}_{(0,0)}$. Hence

$$
D_{m, n} \cong D_{m, 0} \text {. }
$$

If we take in particular $D_{m, 0}=D_{\mathrm{b}}$ and $D_{0,1}=D_{\mathrm{a}}$ we get

$$
D_{\mathrm{b}+n \mathbf{a}} \cong D_{\mathbf{b}} \text {, q.e.d. }
$$

$\mathbf{X}$ - 5. Theorem. Assume we have a field theory containing $n$ fields such that the representations of $\mathfrak{A}$ are characterized by a lattice $L$. 
Then there exists a maximal sublattice $L_{1} \subset L$ such that $D_{\mathbf{a}+\mathbf{a}_{1}} \cong D_{\mathbf{a}}$ for all $\mathbf{a} \in L$ and $\mathbf{a}_{1} \subset L_{1}$.

Every degeneracy which appears in $L$ is of this form. In particular, if there are no degeneracies in $L$ we have $L_{1}=\mathbf{0}$.

Proof. It is clear from Proposition X -1 and Lemma X -4 that all representations which are equivalent to the representation $D_{0}$ form a sublattice. Hence, by Lemma X -4 the equation

$$
D_{\mathrm{a}+\mathrm{a}_{1}} \cong D_{\mathrm{a}}
$$

holds for all $\mathbf{a} \in L$ and $\mathrm{a}_{1} \in L_{1}$. Now Lemma $\mathrm{X}-2$ and 3 imply that every degeneracy is of this form.

We want now to consider the case where $L_{1}$ is not trivial. From the basis theorem for an Abelian group with a finite number of generators, it follows that we can find $n$ linearly independent elements $\mathbf{a}_{1} \ldots \mathbf{a}_{n}$ such that these generate $L$ and moreover $k_{1} \mathbf{a}_{1}, k_{2} \mathbf{a}_{2}, \ldots, k_{n} \mathbf{a}_{n}$ generate $L_{1}$ (some of the $k$ 's might be zero).

$\mathbf{X}$ - 6. Definition. We define $\chi_{j}$ to be the unitary operator which maps $\mathfrak{G}_{\mathbf{b}}$ onto $\mathfrak{S}_{\mathfrak{b}-k_{j} \mathbf{a}_{j}}$ and which establishes the unitary equivalence between the representations $D_{\mathbf{b}}$ and $D_{\mathbf{b}-k_{j} \mathbf{a}_{j}}$

$$
\chi_{j} D(\mathfrak{Q})=D(\mathfrak{A}) \chi_{j} .
$$

$\chi_{j}$ restricted to $\mathfrak{S}_{\mathrm{b}}$ is uniquely defined up to a phase-factor.

$\mathbf{X}-$ 7. Lemma. The arbitrary phase-factors in the definition of $\chi_{j}$ can be adjusted such that $\chi_{j}$ commutes also with the fields $\psi_{i} i=1 \ldots n$.

Proof. Let us consider an expression of the form

$$
P_{\mathbf{b}} \psi^{* k} \chi_{j}^{-1} \psi^{k} \chi_{j} P_{\mathbf{b}} \text {. }
$$

Since $\psi^{\prime k}=D(U) \psi^{k}$ for $U \in \mathfrak{A}$, we see that this expression is independent of the special choice of $\psi$ and hence commutes with every element of $D_{\mathbf{b}}(\mathfrak{Q})$. This means it is equal to a number

Now we get

$$
\lambda(\mathbf{b},-\mathbf{k}) \text { with }|\lambda|=1 .
$$

$$
\begin{gathered}
P_{\mathbf{b}} \psi^{* \mathrm{k}_{1}} \psi^{* \mathrm{k}_{2}} \chi_{j}^{-1} \psi^{\mathrm{k}_{2}} \psi^{\mathrm{k}_{1}} \chi_{j} P_{\mathbf{b}}=\lambda\left(\mathbf{b},-\mathbf{k}_{1}-\mathbf{k}_{2}\right) \\
=P_{\mathbf{b}} \psi^{* \mathbf{k}_{1}} \psi^{* \mathrm{k}_{2}} \chi_{j}^{-1} \psi^{\mathbf{k}_{2}} \chi_{j} \chi_{j}^{-1} \psi^{\mathbf{k}_{1}} \chi_{j} P_{\mathbf{b}}=\lambda\left(\mathbf{b}-\mathbf{k}_{1},-\mathbf{k}_{2}\right) \lambda\left(\mathbf{b},-\mathbf{k}_{1}\right) .
\end{gathered}
$$

This equation implies

Define now

$$
\lambda(\mathbf{b}, \mathbf{k})=\lambda(\mathbf{0}, \mathbf{b}+\mathbf{k}) \bar{\lambda}(0, \mathbf{b}) .
$$

then we get

$$
\chi_{j}^{\prime} P_{\mathrm{b}}=\lambda(0, \mathrm{~b}) \chi_{j} P_{\mathrm{b}} ;
$$

$$
\begin{aligned}
P_{\mathrm{b}} \psi^{* k} \chi_{j}^{\prime-1} \psi^{k} \chi_{j} P_{\mathbf{b}} & =\lambda(\mathbf{0}, \mathbf{b}) \bar{\lambda}(\mathbf{0}, \mathbf{b}-\mathbf{k}) P_{\mathbf{b}} \psi^{* k} \chi_{j}^{-1} \psi^{k} \chi_{j} P_{\mathbf{b}} \\
& =\lambda(\mathbf{0}, \mathbf{b}) \lambda(\mathbf{0}, \mathbf{b}-\mathbf{k}) \bar{\lambda}(\mathbf{b},-\mathbf{k})=1 .
\end{aligned}
$$

This proves our Lemma. 
$\mathbf{X}-8$. Lemma. Let the operators $\chi_{j}$ be such that they commute with all elements of $\mathscr{A}$ and the $\psi$ 's; then the $\chi_{j}$ commute with each other and moreover $\chi_{j}^{-1}\left(\psi^{\mathbf{a}_{j}}\right)^{k_{j}}$ is an element of $\mathfrak{A}$ if we assume that the $\psi$ 's obey the normal commutation relations.

Proof. We first prove the second statement. From the definition of $\chi_{j}$ follows

$$
\chi_{j}^{-1}\left(\psi^{\mathbf{a}_{j}}\right)^{k_{j}} \mathfrak{G}_{\mathrm{b}}=\mathfrak{G}_{\mathbf{b}} .
$$

Let now $\psi_{i} \in \mathfrak{F}_{i}(\mathcal{O})$; then we have for $X \in D_{\mathbf{b}}(\mathfrak{Q}(\mathcal{O}))^{\prime}$

$$
\chi_{j}^{-1}\left(\psi^{\mathbf{a}_{j}}\right)^{k_{j}} P_{\mathbf{b}} X=X \chi_{j}^{-1}\left(\psi^{\mathbf{a}_{j}}\right)^{k_{j}} P_{\mathbf{b}} .
$$

Hence $\chi_{j}^{-1}\left(\psi^{\mathbf{a}_{j}}\right)^{k_{j}} P_{\mathbf{b}}=D_{\mathbf{b}}\left(U_{\mathbf{b}}\right)$, with $U_{\mathbf{b}} \in \mathfrak{A}(\mathcal{O})$.

Let now $\mathcal{O}_{1}$ be spacelike separated from $\mathcal{O}$ and $\psi_{i}^{\prime} \in \mathfrak{F}_{i}\left(\mathcal{O}_{1}\right)$; then we get

$$
\begin{gathered}
\chi_{j}^{-1}\left(\psi^{\left.\mathbf{a}_{j}\right)^{k_{j}}} P_{\mathbf{b}} \psi^{\prime \mathbf{l}}=\chi_{j}^{-1}\left(\psi^{\mathbf{a}_{j}}\right)^{k_{j}} \psi^{\prime 1} P_{\mathbf{b}+1}\right. \\
=\varepsilon\left(k_{j} \mathbf{a}_{j}, \mathbf{l}\right) \psi^{\prime \mathbf{l}} \chi_{j}^{-1}\left(\psi^{\mathbf{a}_{j}}\right)^{k_{j}} P_{\mathbf{b}+\mathbf{l}}=\varepsilon\left(k_{j} \mathbf{a}_{j}, \mathbf{l}\right) \psi^{\prime \mathbf{l}} D_{\mathbf{b}+\mathbf{l}}\left(U_{\mathbf{b}+\mathbf{l}}\right) .
\end{gathered}
$$

On the other hand we have

$$
\chi_{j}^{-1}\left(\psi^{\mathbf{a}_{j}}\right)^{\mathbf{k}_{j}} P_{\mathbf{b}} \psi^{\prime \mathbf{k}}=D_{\mathbf{b}}\left(U_{\mathbf{b}}\right) \psi^{\prime \mathbf{l}}=\psi^{\prime \mathbf{l}} D_{\mathbf{b}+\mathbf{l}}\left(U_{\mathbf{b}}\right) .
$$

But this implies

where

$$
U_{\mathbf{b}}=\varepsilon\left(k_{j} \mathbf{a}_{j}, \mathbf{l}\right) U_{\mathbf{b}+\mathbf{l}}
$$

$$
\varepsilon\left(k_{j} \mathbf{a}_{j}, \mathbf{l}\right)=\left(\psi^{\mathbf{a}_{j}}\right)^{k_{j} *} \psi^{\prime \mathbf{l}^{*}}\left(\psi^{\mathbf{a}_{j}}\right)^{k_{j}} \psi^{\prime \mathbf{l}} .
$$

We consider now the case $\psi^{\prime 1}=\left(\psi^{\prime a_{j}}\right)^{k_{j}}$; then we get

$$
\begin{aligned}
\chi_{j}^{-1}\left(\psi^{\mathbf{a}_{\mathbf{j}}}\right)^{k_{j}} \chi_{j}^{-1}\left(\psi^{\prime} \mathbf{a}_{j}\right)^{k_{j}} P_{\mathbf{b}} & =D\left(U_{\mathbf{b}} \cdot U_{\mathbf{b}}^{\prime}\right) P_{\mathbf{b}} \\
& =D\left(U_{\mathbf{b}}^{\prime} \cdot U_{\mathbf{b}}\right) P_{\mathbf{b}} .
\end{aligned}
$$

On the other hand we get

$$
\begin{aligned}
\chi_{j}^{-1}\left(\psi^{\mathbf{a}_{j}}\right)^{k_{j}} \chi_{j}^{-1}\left(\psi^{\prime \mathbf{a}_{j}}\right)^{k_{j}} P_{\mathbf{b}} & =\varepsilon\left(k_{j} \mathbf{a}_{j}, k_{j} \mathbf{a}_{j}\right) \chi_{j}^{-1}\left(\psi^{\prime \mathbf{a}_{j}}\right)^{k_{j}} \chi_{j}^{-1}\left(\psi^{\mathbf{a}_{j}}\right)^{k_{j}} P_{\mathbf{b}} \\
& =\varepsilon\left(k_{j} \mathbf{a}_{j}, k_{j} \mathbf{a}_{j}\right) D\left(U_{\mathbf{b}}^{\prime}, U_{\mathbf{b}}\right) P_{\mathbf{b}} .
\end{aligned}
$$

From this follows $\varepsilon\left(k_{j} \mathbf{a}_{j}, k_{j} \mathbf{a}_{j}\right)=1$, and since we have assumed normal commutation relations we get $\varepsilon\left(k_{j} \mathbf{a}_{j}, \mathbf{l}\right)=1$. Hence $U_{\mathbf{b}}$ is independent of $\mathbf{b}$.

Now we get

and from this

$$
\begin{aligned}
\chi_{i} \chi_{j}^{-1}\left(\psi^{\mathbf{a}_{j}}\right)^{k_{j}} & =\chi_{i} D(U)=D(U) \chi_{i} \\
& =\chi_{j}^{-1}\left(\psi^{\mathbf{a}_{j}}\right)^{k_{j}} \chi_{i}=\chi_{j}^{-1} \chi_{i}\left(\psi^{\mathbf{a}_{j}}\right)^{k_{j}}
\end{aligned}
$$

This proves the lemma.

$$
\chi_{i} \chi_{j}=\chi_{j} \chi_{i}
$$

$\mathbf{X}-9$. Theorem. Assume we have given $n+1$ representations $D_{i}$ of $\mathfrak{A}$. Let us denote by $L$ the lattice of representations we get by the normal way of construction. Assume there exists a sublattice $L_{1} \subset L$ of 
representations which are all equivalent to the vacuum representation $D_{0}$. Then there exist "charged fields" $\phi_{i} i=1,2, \ldots, m \leqq n$ and numbers $k_{i}$ such that

1) $\phi_{i}^{k_{i}} \in \mathfrak{A}$.

2) All subrepresentations of $\mathfrak{A}$ are labeled by elements of $L / L_{1}$ and no two of these representations are equivalent.

3) To every representation in $L$ there exists a representation in $L / L_{1}$ which is equivalent to this representation.

Proof. We define operators $\phi_{i}$ by the relation

$$
\phi_{i} \mathfrak{G}_{\Sigma l_{j} \mathbf{a}_{j}}=\left\{\begin{array}{llll}
\psi^{\mathbf{a}_{j}} \mathfrak{G}_{\Sigma l_{j} \mathbf{a}_{j}} & \text { if } & l_{i} \equiv 1 & \bmod k_{i} \\
\chi_{i}^{-1} \psi^{\mathbf{a}_{i}} \mathfrak{G}_{\Sigma l_{j} \mathbf{a}_{j}} & \text { if } & l_{i} \equiv 1 & \bmod k_{i} .
\end{array}\right.
$$

From this definition together with Lemma $\mathrm{X}-8$ follows $\phi_{i}^{k_{i}} \in \mathfrak{A}$.

Let now $\mathcal{O}_{1}$ and $\mathcal{O}_{2}$ be spacelike separated and $\phi_{i} \in \mathfrak{F}_{i}\left(\mathcal{O}_{1}\right)$ and $a \in \mathfrak{A}\left(\mathcal{O}_{2}\right)$; then we get

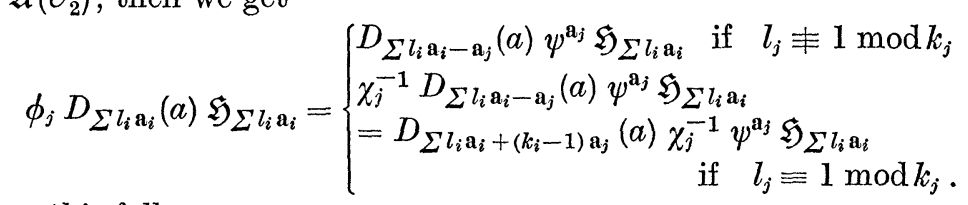

From this follows

$$
\phi_{j} D(a)=D(a) \phi_{j} .
$$

Now assume $\phi_{i} \in \mathfrak{F}_{i}\left(\mathcal{O}_{1}\right)$ and $\phi_{k} \in \mathfrak{F}_{k}\left(\mathcal{O}_{2}\right)$; then we get, if $j \neq i$,

and hence

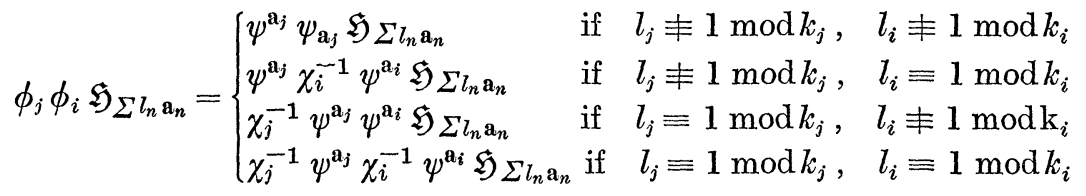

$$
\begin{aligned}
& =\varepsilon\left(\mathbf{a}_{j} \mathbf{a}_{i}\right) \phi_{i} \phi_{j} \mathfrak{G} \Sigma l_{n} \mathbf{a}_{n} .
\end{aligned}
$$

If $j=i$ we get

$$
\phi_{j} \phi_{i}=\varepsilon\left(\mathbf{a}_{j}, \mathbf{a}_{i}\right) \phi_{i} \phi_{j} .
$$

$$
\begin{aligned}
\phi_{j} \phi_{j}^{\prime} \mathfrak{S}_{\Sigma l_{n} \mathbf{a}_{n}} & = \begin{cases}\psi^{\mathbf{a}_{j}} \psi^{\prime \mathbf{a}_{j}} \mathfrak{G} \sum_{l_{n} \mathbf{a}_{n}} & \text { if } l_{j} \neq 1,2 \bmod k_{j} \\
\psi^{\mathbf{a}_{j}} \chi_{j}^{-1} \psi^{\prime \mathbf{a}_{j}} \mathfrak{G} \Sigma l_{n} \mathbf{a}_{n} & \text { if } l_{j} \equiv 1 \quad \bmod k_{j} \\
\chi_{j}^{-1} \psi^{\mathbf{a}_{j}} \psi^{\prime \mathbf{a}_{j}} \mathfrak{G} \Sigma l_{n} \mathbf{a}_{n} & \text { if } l_{j} \equiv 2 \quad \bmod k_{j}\end{cases} \\
& =\varepsilon\left(\mathbf{a}_{j}, \mathbf{a}_{j}\right) \phi_{j}^{\prime} \phi_{j} \mathfrak{G} \Sigma l_{n} \mathbf{a}_{n},
\end{aligned}
$$

and hence

$$
\phi_{j} \phi_{j}^{\prime}=\varepsilon\left(\mathbf{a}_{j}, \mathbf{a}_{j}\right) \phi_{j}^{\prime} \phi_{j},
$$

where

$$
\varepsilon\left(\mathbf{a}_{j}, \mathbf{a}_{i}\right)=\psi^{\mathbf{a}_{j} *} \psi^{\mathbf{a}_{i} *} \psi^{\mathbf{a}_{j}} \psi^{\mathbf{a}_{i}} .
$$

This means the new fields fulfil the same commutation relations as the fields $\psi^{\mathbf{a}_{i}}$. 
We apply now the algebra generated by $D(\mathfrak{A})$ and the $\phi$ 's to the original vacuum state. This is an invariant subspace and is of the form

This proves our theorem.

$$
\sum_{\substack{l_{i}=-\infty \\ k_{i}=0}}^{+\infty} \sum_{\substack{l_{i}=0 \\ k_{j}=0}}^{k_{j}-1} \mathfrak{S} \Sigma l_{i} \mathbf{a}_{i} .
$$

Acknowledgements. The author would like to thank G. F. Dell'Antonro for many discussions.

\section{References}

[1] Jordan, P., u. E. Wigner: Z. Phys. 47, 631 (1928).

[2] SCHWINGER, J.: Selected papers on quantum electrodynamics. New York: Dover Publications, Inc. 1958.

[3] Pauli, W.: Phys. Rev. 58, 716 (1940).

[4] Streater, R. F., and A. S. Wightman: PCT, Spin and Statistics, and All That. New York: W. A. Benjamin Inc. 1964.

[5] Greenberg, O. W., and A. M. L. Messiah: Phys. Rev. 136, B248 (1964).

[6] Green, H. S.: Phys. Rev. 90, 270 (1953).

[7] Borchers, H. J.: Commun. math. Phys 1, 57 (1965).

[8] Namark, M. A.: Normed Rings. Groningen: P. Nordhoff 1959.

[9] Araki, H.: Progress Theor. Phys 32, 844 (1964). Borchers, H. J.: Commun. math. Phys. 1, 49 (1965).

[10] Wightman, A. S.: Trieste Lectures (1962), Theorem 12.

[11] Dunford, N., and J.T. Schwartz: Linear Operators, Part I, p. 132. New York: Interscience Publishers 1958.

[12] Jost, R., e H. Lehmann: Nuovo Cimento 5, 1598 (1957).

F. J. Dyson: Phys. Rev. 110, 1460 (1958).

[13] Araki, H.: J. Math. Phys. 2, 267 (1961).

(Received June 1, 1965) 